\title{
Field theory configuration spaces for connective ko-theory
}

\author{
ELKE K MARKERT
}

\begin{abstract}
We describe a new $\Omega$-spectrum for connective ko-theory formed from spaces inf ${ }_{n}$ of operators which have certain nice spectral properties, and which fulfill a connectivity condition. The spectral data of such operators can equivalently be described by certain Clifford-linear, symmetric configurations on the real axis; in this sense, our model for ko stands between an older one by Segal [20], who uses nonsymmetric configurations without Clifford-structure on spheres, and the well-known Atiyah-Singer model for KO using Clifford-linear Fredholm operators [1]. Dropping the connectivity condition we obtain operator spaces $\operatorname{Inf}_{n}$. These are homotopy equivalent to the spaces $\mathcal{E} \mathcal{F} \mathcal{T}_{n}$ of $1 \mid 1$-dimensional supersymmetric Euclidean field theories of degree $n$ which were defined by Stolz and Teichner in [22; 23] and with Hohnhold in [9] (in terms of certain homomorphisms of super semigroups). They showed that the $\mathcal{E} \mathcal{F} \mathcal{T}_{-n}$ are homotopy equivalent to $\mathrm{KO}_{n}$ and gave the idea for the connection between $\mathcal{E} \mathcal{F} \mathcal{T}_{n}$ and $\operatorname{Inf}_{n}$. We can derive a homotopy equivalent version of the $\Omega$-spectrum inf in terms of "field theory type" super semigroup homomorphisms. Tracing back our connectivity condition to the functorial language of field theories provides a candidate for connective 1|1-dimensional Euclidean field theories, eft, and might result in a more general criterion for instance for a connective version of 2|1-dimensional such theories (which are conjectured to yield a spectrum for TMF).
\end{abstract}

19L41, 55N15, 81Q60; 81T60, 81T08

\section{Introduction}

Our main motivation for this paper comes out of a project of Stolz and Teichnerpublished works include $[22 ; 23]$ as well as work with Hohnhold (referred to as Hohnhold et al) [9]—who define 1|1-dimensional and 2|1-dimensional supersymmetric Euclidean field theories as functors on certain (super) geometric bordism categories in order to obtain new geometric models for $K$-theory and the "universal elliptic theory" TMF of topological modular forms, respectively. This approach was initiated by Segal [21] and Witten [25]. The less difficult field theory model of $K$-theory should be considered a case study for 2|1-dimensional field theories and TMF. In this simpler case, Stolz and Teichner define spaces $\mathcal{S G O}_{n}, n \in \mathbb{Z}$, of homomorphisms of certain generalized super semigroups, or super semigroups of operators. Subspaces of 
these homomorphism spaces correspond directly to 1|1-dimensional, supersymmetric Euclidean field theories (EFTs) of degree $n$ in the sense that the homomorphisms correspond to the evaluation of the field theory functors on a particular elementary super moduli space (the super moduli space of super intervals). One can therefore speak of a space $\mathrm{EFT}_{n}$ of such field theories, namely the set with the topology inherited from the subspace of SGOs. They show that the subspaces are homotopy equivalent to $\mathcal{S G O} \mathcal{O}_{n}$ themselves, and the latter form an $\Omega$-spectrum for KO.

In the original paper by Stolz and Teichner [22], super semigroups of operators satisfying a certain Hilbert-Schmidt condition are derived from the functorial definition of degree $n$ EFTs which is postulated there; the authors conclude that such a field theory is already completely determined by this data. This determines a subspace $\mathcal{S G} \mathcal{O}_{n}^{\mathrm{HS}}$ which is homotopy equivalent to $\mathcal{S G O}_{n}$. Since [22], a lot of work has been done adjusting the definitions of field theory functors; the main references so far are Stolz and Teichner [23] and Hohnhold, Stolz and Teichner [9]. These are still incomplete concerning the definition of degrees of field theories, but the constructions have been outlined by Stolz and Teichner and are expected to appear in literature in the near future. We will not attempt to include any descriptions of the functorial aspects here, however we note that for 1|1-dimensional Euclidean field theories the results remain basically unchanged: using the new definitions of [23; 9], one obtains again spaces of field theories, $\mathrm{EFT}_{n}$, topologized as subspaces $\mathcal{S G O}_{n}^{\mathrm{TC}}$ of $\mathcal{S G O} \mathcal{G}_{n}$ satisfying a trace-class condition. These slightly different subspaces are again homotopy equivalent to $\mathcal{S G O}_{n}$; see Hohnhold et al [9]. For the moment one can thus think of such super semigroups of operators as defining EFTs.

The spaces $\mathcal{S G O}_{n}$ themselves are quite well understood. In particular, elements in $\mathcal{S G O}_{n}$ have unique infinitesimal generators: these are Clifford-linear operators on a Hilbert space $\mathcal{H}_{n}$ with $\mathcal{C}_{n}$-module structure. This was done in $[22 ; 8]$. The operators are in general unbounded, but they do have relatively nice spectral properties (for example discrete, real spectra). One obtains homeomorphisms between $\mathcal{S G O}_{n}$ and spaces of such operators $\operatorname{Inf}_{n}$. The operator spaces $\operatorname{Inf}_{n}$ are comparatively easy to deal with; in fact, there are several equivalent descriptions of them in terms of configurations, or $\mathcal{C}^{*}$-algebra homomorphisms. Configuration spaces, which basically describe the spectral data of the operators in $\operatorname{Inf}_{n}$, provide a very convenient graphic model. The description in terms of $\mathcal{C}^{*}$-algebra homomorphisms and a model of $\mathrm{KO}$ in this language (see Higson and Guenthner [7] and Joachim [11]) is used in the original work by Stolz and Teichner [22] to prove the result on the homotopy type of $\mathrm{EFT}_{n}$. The result is shown again in Hohnhold et al [9] for the more recent definitions of EFTs; here the authors employ configuration spaces to connect the spaces $\operatorname{Inf}_{n}, \mathcal{S G O}_{n}$ and $\mathrm{EFT}_{n}$ 
(the latter discussed only for degree 0) with other well-known models of KO such as Fredholm operators.

In this paper we give a connectivity condition and provide Inf with a connective version inf. To be precise, we define connective covers $\operatorname{Inf}_{n}^{k} \sim \operatorname{Inf}_{n}\langle k\rangle$ of the spaces in question and set $\inf _{-n}:=\operatorname{Inf}_{-n}^{n}$. This is a new $\Omega$-spectrum for ko, which also comes equipped with the structure maps of an $\Omega$-spectrum (the proofs in [22] and [9] for the periodic case do not provide such maps explicitly). We work it out in terms of operator spaces and configuration spaces, the condition can however be expressed strictly in terms of super semigroups of operators, thus producing the connective version sgo.

In fact, the construction also provides an idea how to reformulate the definition of $s g o$ for field theories, that is, for 1|1-EFT functors, to obtain a connective spectrum eft (see Remark 2.5). Moreover, this seems to be independent of the dimension and might be useful as well to define "connective" $d \mid 1-$ EFT functors. We hope to be able to specify this in the future.

We will start out with the definition of the spaces $\mathcal{S G \mathcal { G } _ { n }}$ of super semigroup homomorphisms and the theorem of Stolz and Teichner relating them to KO. To do that we explain shortly some of the terminology, before proceeding to define the connective spaces sgo. In Section 3 we turn to generating operators and configurations and define their connective versions. The proof of connectivity and spectrum properties follows in Section 4. It is done by constructing quasifibrations with contractible total spaces (this proof was inspired by a proof of Bott periodicity by M Behrens [2]).

Acknowledgements This work is based on my doctoral thesis [13] written under the supervision of S Stolz at the University of Notre Dame in 2005. I would like to thank my advisor S Stolz and the faculty at Notre Dame, in particular W Dwyer, L Taylor and B Williams, for their teaching and their constant support. I would like to thank F Dumitrescu, H Hohnhold, C Redden and particularly G Gaudens for so many helpful comments, questions and discussions.

\section{Super semigroups of operators}

We begin with a short summary of the relevant definitions and facts from the theory of super manifolds and super groups (we refer the interested reader to Deligne and Morgan [4] and Varadarajan [24]).

The category of supermanifolds $\mathcal{S M}$ has as its objects ringed spaces $\mathcal{M}^{m \mid n}:=$ $\left(|\mathcal{M}|, \mathcal{O}_{\mathcal{M}}\right)$ where $|\mathcal{M}|$ is an $m$-manifold and $\mathcal{O}_{\mathcal{M}}$ is a sheaf of $\mathbb{Z} / 2$-graded commutative algebras, locally (in $|\mathcal{M}|$ ) isomorphic to the sheaf $\mathcal{C}^{\infty}(-) \otimes \Lambda \mathbb{R}^{n}$, where $\Lambda \mathbb{R}^{n}$ 
denotes the exterior algebra of $\mathbb{R}^{n}$. Morphisms are morphisms of ringed spaces. The fundamental examples are the supermanifolds $\mathbb{R}^{m \mid n}:=\left(\mathbb{R}^{m}, \mathcal{O}_{\mathbb{R}^{m \mid n}}\right)$ where $\mathcal{O}_{\mathbb{R}^{m \mid n}}(U):=\mathcal{C}^{\infty}(U) \otimes \Lambda \mathbb{R}^{n}$. Supermanifolds are locally isomorphic to these. For convenience in calculating and describing supermanifolds and their morphisms one often makes use of the so-called functor of points approach. This is nothing but an application of Yoneda's lemma to $\mathcal{S M}$, by which there is an inclusion of $\mathcal{S M}$ as a full subcategory into the category of functors and natural transformations $\mathcal{F} u n(\mathcal{S M}$ op Sets $)=: \mathcal{G S M}$

$$
\mathcal{S M} \rightarrow \mathcal{G S M}, \quad \mathcal{M} \mapsto(\mathcal{S} \mapsto \mathcal{S} \mathbb{M}(\mathcal{S}, \mathcal{M})) .
$$

We call an object in $\mathcal{G S M}$ a generalized supermanifold; the set $\mathcal{S} \mathbb{M}(\mathcal{S}, \mathcal{M})=: \mathcal{M}(\mathcal{S})$ is called the set of $\mathcal{S}$-points of the supermanifold $\mathcal{M}$. In terms of $\mathcal{S}$-points we have local coordinates of a supermanifold given by the isomorphisms

$$
\mathbb{R}^{m \mid n}(\mathcal{S}) \cong\left\{\left(t_{1}, \ldots, t_{m} ; \theta_{1}, \ldots, \theta_{n}\right) \mid t_{i} \in\left(\Gamma^{\infty} \mathcal{O}_{\mathcal{S}}\right)^{\mathrm{ev}}, \theta_{j} \in\left(\Gamma^{\infty} \mathcal{O}_{\mathcal{S}}\right)^{\text {odd }}\right\} .
$$

One can also make sense of geometric constructions like tangent and cotangent bundles, differential forms, super Lie groups and super Lie algebras, in a fashion quite analogous to the nonsuper situation; see Deligne and Morgan [4]. In particular, a super Lie group $\mathcal{G}$ is a group object in the category $\mathcal{S} \mathbb{M}$, and its multiplication can be described in terms of $\mathcal{S}$-points as a map

$$
\mathcal{G}(\mathcal{S}) \times \mathcal{G}(\mathcal{S}) \rightarrow \mathcal{G}(\mathcal{S})
$$

which is functorial in $\mathcal{S}$. Similarly, we can describe super semigroups, and generalized versions of both. The examples important to us are the following.

Example Let $V=V^{0} \oplus V^{1}$ be a $\mathbb{Z} / 2$-graded Banach space (possibly infinitedimensional). Then we define a generalized supermanifold by determining its values on opens in $\mathbb{R}^{m \mid n}, m, n \in \mathbf{N}$, ie $\mathcal{U}:=\left(|\mathcal{U}|, \mathcal{C}^{\infty}(|\mathcal{U}|)\left[\theta_{1}, \ldots, \theta_{n}\right]\right) \subset \mathbb{R}^{m \mid n}$. On these local models we set

$$
\mathcal{V}: \mathcal{U} \mapsto\left(\mathcal{C}^{\infty}(|\mathcal{U}| ; V)\left[\theta_{1}, \ldots, \theta_{n}\right]\right)^{\mathrm{ev}}
$$

where the latter denotes the set of even elements. To turn this into a functor on the category of supermanifolds, we define the functor on maps of such opens by Taylor expansion (compare Deligne and Morgan [4] and Hohnhold et al [9]) and use gluing to extend it to all supermanifolds. If $V \cong \mathbb{R}^{m} \oplus \mathbb{R}^{n}$ is finite-dimensional, this is the functor representing $\mathbb{R}^{m \mid n}$. We will apply this formalism to the algebra of bounded operators on some graded Hilbert space. This produces a generalized super semigroup, where the multiplication comes from multiplication of operators.

Example The following defines a super group structure on $\mathbb{R}^{1 \mid 1}$ :

$$
\mathbb{R}^{1 \mid 1}(\mathcal{S}) \times \mathbb{R}^{1 \mid 1}(\mathcal{S}) \rightarrow \mathbb{R}^{1 \mid 1}(\mathcal{S}), \quad(t, \theta) \circ(s, \eta) \mapsto(t+s+\theta \eta, \theta+\eta) .
$$


It is easy to check that this defines in fact a group structure on $\mathbb{R}^{1 \mid 1}(\mathcal{S})$. We sometimes refer to this as the "twisted" super group structure. Note that we can restrict it to $\mathbb{R}_{>0}^{1 \mid 1}:=\left(\mathbb{R}_{>0},\left.\mathcal{O}_{\mathbb{R}^{1 \mid 1}}\right|_{\mathbb{R}_{>0}}\right)$, the subsupermanifold whose reduced part is the positive real axis. The twisted super group structure makes $\mathbb{R}_{>0}^{1 \mid 1}$ into a super semigroup.

Let us now define the Hilbert universes mentioned in the introduction. We denote by $\mathcal{C}_{n}, n \geqslant 0$, the standard real Clifford algebra on generators $e_{1}, \ldots, e_{n}$ where $e_{i} e_{j}+e_{j} e_{i}=-2 \delta_{i j}$. For negative $n, \mathcal{C}_{n}$ denotes the real Clifford algebra with respect to the negative definite inner product, ie with generators which anticommute pairwise and square to +1 .

In the following we are going to work with Hilbert spaces which come with $\mathcal{C}_{n}$-module structures. We call such a structure compatible with the inner product on the Hilbert space, if the generators of $\mathcal{C}_{n}\left(\mathcal{C}_{-n}\right.$, respectively) act as skew-adjoint (self-adjoint) operators. This implies in particular that orthogonal projections onto submodules along the given inner product in the Hilbert space are linear with respect to the Clifford algebra action. Clifford algebras are graded into even and odd elements; we distinguish graded and ungraded modules over them. It is a well-known fact (see for example Lawson and Michelson [12]) that the even part $\mathcal{C}_{n}^{0}$ of $\mathcal{C}_{n}$ is isomorphic to $\mathcal{C}_{n-1}$ as ungraded algebras. We use this in the definition below to obtain a graded $\mathcal{C}_{n}$-module from an ungraded $\mathcal{C}_{n-1}$-module. We will further use right and left module structures; note here that one can consider a right $\mathcal{C}_{n}$-module structure as left $\mathcal{C}_{-n}$ structure by replacing the generators $e_{1}, \ldots, e_{n}$ of $\mathcal{C}_{n}$ acting from the right with generators $\epsilon e_{1}, \ldots, \epsilon e_{n}$ of $\mathcal{C}_{-n}$ acting from the left ( $\epsilon$ denotes the grading involution). We will distinguish these actions also in the names of the generators; we will denote generators acting from the left by $f_{i}$, those acting from the right by $e_{i}$. In particular, we will often consider Hilbert spaces equipped with a $\mathcal{C}_{n+k}$-right module structure as bimodules over $\mathcal{C}_{-k}-\mathcal{C}_{n}$. This is then expressed accordingly in the notation of the generators acting on it: we set $f_{i}:=\epsilon e_{i}, i=n+1, \ldots, n+k$.

Definition 2.1 For each $n \in \mathbb{Z}$, we fix an infinite-dimensional separable real Hilbert space $H_{n}$ with compatible (ungraded) right $\mathcal{C}_{n-1}$-module structure, which contains all irreducible $\mathcal{C}_{n-1}$-modules infinitely many times. We then set $\mathcal{H}_{n}:=H_{n} \otimes_{\mathcal{C}_{n-1}} \mathcal{C}_{n}$. This is a $\mathbb{Z} / 2$-graded real Hilbert space (grading involution denoted by $\epsilon$ ) with compatible $\mathcal{C}_{n}$-module structure, which contains all irreducible graded $\mathcal{C}_{n}$-modules infinitely many times.

We further denote by $\mathcal{B}_{\mathcal{C}_{n}}\left(\mathcal{H}_{n}\right)$ the algebra of bounded, Clifford-linear operators on $\mathcal{H}_{n}$, equipped with the norm topology. This is an infinite-dimensional $\mathbb{Z} / 2$-graded Banach algebra (the grading into even and odd operators is induced by the grading on $\mathcal{H}_{n}$ ). 
Recall that this can be interpreted as generalized super group by the functor from the first example. As in [9], we define a super semigroup of operators of degree $n$ as a homomorphism of generalized super semigroups

$$
\phi \in \operatorname{Hom}_{\mathrm{sgg}}\left(\mathbb{R}_{>0}^{1 \mid 1}, \mathcal{B}_{\mathcal{C}_{n}}\left(\mathcal{H}_{n}\right)\right),
$$

where $\mathbb{R}_{>0}^{1 \mid 1}$ carries the twisted super semigroup structure. We define a topology on this Hom-set by evaluating a homomorphism on the universal element id $\in \mathbb{R}_{>0}^{1 \mid 1}\left(\mathbb{R}_{>0}^{1 \mid 1}\right)$. We get two families of operators $t \mapsto A(t)=e^{-t G_{E}^{2}}, t \mapsto B(t)=e^{-t G_{E}^{2} G_{E}}$ for $t \in \mathbb{R}_{>0}$. The topology is given by uniform convergence on compact supports in both $A$ and $B$ (see Hohnhold [8, 2.3] and Hohnhold et al [9]).

If a super semigroup of operators takes values in a subset $\mathcal{A} \subset \mathcal{B}_{\mathcal{C}_{n}}\left(\mathcal{H}_{n}\right)$, ie if both its families $A(t)$ and $B(t)$ take values in $\mathcal{A}$, then we write $\phi \in \mathcal{S G O}(\mathcal{A})$ for the resulting subspace of homomorphisms and refer to these as super semigroups of operators with values in $\mathcal{A}$. We set

$$
\mathcal{S G O}_{n}:=\mathcal{S G O}\left(\mathcal{K}_{\mathcal{C}_{n}}\left(\mathcal{H}_{n}\right)\right)
$$

where the latter stands for compact, self-adjoint, $\mathcal{C}_{n}$-linear operators. In the field theory context, there are two other interesting candidates for $\mathcal{A}$, namely $H S_{\mathcal{C}_{n}}^{\mathrm{sa}}\left(\mathcal{H}_{n}\right)$, the subset of self-adjoint Hilbert-Schmidt operators with respect to the Hilbert-Schmidt norm, and $T C_{\mathcal{C}_{n}}^{\text {sa }}\left(\mathcal{H}_{n}\right)$, the subset of self-adjoint trace-class or nuclear operators with respect to the trace or nuclear norm (compare again Hohnhold et al [9]). The corresponding spaces are denoted by $\mathcal{S G \mathcal { O } _ { n } ^ { \mathrm { HS } }}$ and $\mathcal{S G \mathcal { G } _ { n } ^ { \mathrm { TC } }}$, respectively. In the latter reference the authors prove that both inclusions below are homotopy equivalences:

$$
\mathcal{S G O}_{n}^{\mathrm{HS}} \hookrightarrow \mathcal{S G O}_{n} \quad \mathcal{S G O}_{n}^{\mathrm{TC}} \hookrightarrow \mathcal{S G O}_{n}
$$

In the context of infinitesimal generators, or configurations respectively, these homotopy equivalences are quite intuitive (see also Lemma 3.7).

As mentioned in the introduction, the paper [22] contains a preliminary definition of supersymmetric field theories of degree $n$ and an outline for proving a set bijection

$$
\mathrm{EFT}_{n} \leftrightarrow \mathcal{S G O}_{n}^{\mathrm{HS}}
$$

which is then used to topologize $\mathrm{EFT}_{n}$. In the next theorem we collect the corresponding results on the new version which has meanwhile appeared in [23;9], and which provides sound definitions for supersymmetric Euclidean field theories (in dimensions $0 \mid 1$, $1 \mid 1$ and 2|1) in degree 0 . Note that these more recent definitions have not yet been completed for general degree $n$ (this will use the constructions in the original work [22], but will be formulated as twists of field theories of degree 0 ). However we expect the proposed result to hold for all degrees as claimed in the later manuscript [9]. In the 
following, $\mathrm{EFT}_{0}$ refers to the space of 1|1-EFT's of degree 0 over a point as defined in $[23 ; 9]$. The topology on this space is again derived from the one on $\mathcal{S G O}_{0}$. In degree $n$, the claim in (i) below can be taken as a definition, if so wished.

Theorem 2.2 (Hohnhold-Stolz-Teichner) (i) The space $\mathrm{EFT}_{0}$ of supersymmetric $1 \mid 1$-dimensional Euclidean field theories of degree 0 is homeomorphic to $\mathcal{S G O}_{0}^{\mathrm{TC}}$. For integer degree $n$, the correct notion of twisted field theories will produce a homeomorphism

$$
\mathrm{EFT}_{n} \approx \mathcal{S} \mathcal{G} \mathcal{O}_{n}^{\mathrm{TC}}
$$

(ii) A homomorphism $E \in \mathcal{S G O}_{n}$ can be written in the form

$$
E(S): \mathbb{R}_{>0}^{1 \mid 1}(S) \rightarrow \mathcal{B}_{\mathcal{C}_{n}}\left(\mathcal{H}_{n}\right)(S), \quad(t, \theta) \mapsto E(\mathcal{S})(t, \theta):=e^{-t G_{E}^{2}+\theta G_{E}}
$$

where the infinitesimal generator $G_{E}$ of $E$ is a uniquely determined odd $\mathcal{C}_{n}$-linear operator with compact resolvent, defined on some domain $\mathcal{D}\left(G_{E}\right) \subset \mathcal{H}_{n}$. On the orthogonal complement of this domain the operators $E(\mathcal{S})(t, \theta)$ are defined to be zero. In particular, a field theory $E \in \mathrm{EFT}_{n}$ is uniquely determined by its infinitesimal generator $G_{E}$.

(iii) For every $n$, there are weak homotopy equivalences

$$
\mathrm{EFT}_{-n} \sim \mathcal{S} \mathcal{G O}_{-n} \sim \mathrm{KO}_{n}
$$

where the latter is the $n$-th space in an $\Omega$-spectrum associated to KO.

Our main result is that we can express the connective theory ko as well in this language. We obtain a connective $\Omega$-spectrum sgo by constructing connective covers $\mathcal{S G} \mathcal{O}_{n}^{k}$ of the spaces $\mathcal{S G O}_{n}$.

To define $\mathcal{S G O}_{n}^{k}$, note that a homomorphism $E$ of generalized super semigroups in $\mathcal{S G O} \mathcal{O}_{n}$ has a reduced part $|E|:=E\left(\mathbb{R}^{0 \mid 0}\right)$. One should think of the reduced part of a generalized supermanifold $\mathcal{M}$ as $\mathcal{M}\left(\mathbb{R}^{0 \mid 0}\right)$ : in the case of a representable functor this describes the points of the reduced manifold $|\mathcal{M}|$ while in the case of a super semigroup this is a semigroup. It is a semigroup homomorphism of the form

$$
|E|: \mathbb{R}_{>0} \rightarrow \mathcal{B}_{\mathcal{C}_{n}}\left(\mathcal{H}_{n}\right), \quad t \mapsto e^{-t G_{E}^{2}}
$$

and it takes values in $\mathcal{K}_{\mathcal{C}_{n}}\left(\mathcal{H}_{n}\right)$. Let now $\mathrm{SGO}_{n}=\operatorname{SGO}\left(\mathcal{K}_{\mathcal{C}_{n}}\left(\mathcal{H}_{n}\right)\right)$ denote nonsuper semigroups of operators with such values (note here that an element $F \in \mathrm{SGO}_{n}$ is of the form $F(t)=e^{-t A_{F}}$ for some even infinitesimal generator $A_{F}$, which need not have an odd square root; thus it need not be the reduced part of some element in $\left.\mathcal{S G O}_{n}\right)$. Then for $k \geqslant 0$ we define

$$
\mathcal{S G O}_{n}^{k}:=\left\{E \in \mathcal{S G O}\left(\mathcal{K}_{\mathcal{C}_{n}}\left(\mathcal{H}_{n+k}\right)\right)|| E \mid \in \operatorname{SGO}_{n+k}\right\} .
$$


The Hilbert universe $\mathcal{H}_{n+k}$ is a right $\mathcal{C}_{n+k}$-module which we want to think of in the following as $\mathcal{C}_{-k}-\mathcal{C}_{n}$-bimodule (we make this visible by writing $\mathcal{H}_{n}^{k}$ ). The generator $G_{E}$ of an element $E \in \mathcal{S} \mathcal{G} \mathcal{O}_{n}^{k}$ is then an operator on $\mathcal{H}_{n}^{k}$ which is itself only $\mathcal{C}_{n}$-linear, but whose square is $\mathcal{C}_{-k}-\mathcal{C}_{n}$-linear. Now we have:

Theorem 2.3 For $k \geqslant 0$, there are weak homotopy equivalences

$$
\mathcal{S G O}_{n}^{k} \sim \mathcal{S} \mathcal{G} \mathcal{O}_{n}\langle k\rangle
$$

Setting $s g o_{-n}:=\mathcal{S G O} \mathcal{O}_{-n}^{n}$ we obtain:

Theorem 2.4 There are weak homotopy equivalences

$$
s g O_{-n} \sim \mathrm{ko}_{n}
$$

and the spaces $\mathrm{sgo}_{n}$ form an $\Omega$-spectrum for ko.

Remark 2.5 (Connective field theories) (i) In the definition of $\mathcal{S G O}{ }_{n}^{k}$, if we replace compact operators $\mathcal{K}_{\mathcal{C}_{n}}\left(\mathcal{H}_{n+k}\right)$ by trace-class operators $\mathrm{TC}_{\mathcal{C}_{n}}^{\text {sa }}\left(\mathcal{H}_{n+k}\right)$, we obtain spaces $\left(\mathcal{S G} \mathcal{O}_{n}^{k}\right)^{\mathrm{TC}}$. We like to think of these as "connective covers of the field theory spaces $\mathrm{EFT}_{n}^{k}$ ". By the same argument as used for $k=0$ they are homotopy equivalent to $\mathcal{S G O}_{n}^{k}$, and by the same arguments as in this exposition they satisfy

$$
\left(\mathcal{S G O}_{n}^{k}\right)^{\mathrm{TC}} \sim \mathcal{S} \mathcal{G O} \mathcal{O}_{n}^{\mathrm{TC}}\langle k\rangle .
$$

This can be used to define a connective $\Omega$-spectrum eft $:=s g o^{\mathrm{TC}} \sim \operatorname{sgo}$ of "field theory type super semigroups of operators". More important though is the following observation:

(ii) The definition of sgo suggests a similar condition for spaces of EFT functors. The elements of $\mathcal{S G O}_{n}^{\mathrm{TC}}$ correspond to 1|1-dimensional susy EFT's of degree $n$. Now as with super-semi group homomorphisms, we can also obtain from each susy EFT an underlying, or reduced, nonsusy EFT (which will a priori have the same degree as the susy EFT). Then one could define a " $k$-connective susy EFT in degree $n$ " as a susy EFT of degree $n$ whose state space comes with an action of $\mathcal{C}_{n+k}$ and whose underlying, nonsusy EFT is of degree $n+k$. The set $\mathrm{EFT}_{n}^{k}$ of $1 \mid 1-$ dimensional $k$-connective susy EFT's should then correspond bijectively to $\left(\mathcal{S G O}_{n}^{k}\right)^{\mathrm{TC}}$ in the same manner as for nonconnective EFT's. Working this out would produce the honest version eft $\approx s g o^{\mathrm{TC}}$.

Next we give more convenient descriptions of the spaces $\mathcal{S G O}{ }_{n}^{k}$ in terms of operators and configurations. 


\section{Configurations and operators}

Let $\mathcal{G r}(H)$ denote the Grassmannian of finite-dimensional linear subspaces of an infinite-dimensional Hilbert space $H$. A subspace in $\mathcal{G} r(H)$ corresponds to the orthogonal projection operator onto it, in this way the Grassmannian is equivalent to the finite-rank projection operators on $H$. We equip it with the norm topology.

Definition 3.1 Let $X$ be a compact topological Hausdorff space with basepoint $*$, such that there is a homotopy on $X$ which strongly retracts a neighbourhood of the basepoint to $*$ (in slight abuse of notation we refer to this property as being "wellpointed"). Let $H$ be an infinite-dimensional Hilbert space. Then a configuration on $X$ with labels (or coefficients) in $H$ is a map

$$
c:(X-*) \rightarrow \mathcal{G r}(H)
$$

where

- $\sigma(c):=\{x \in(X-*) \mid c(x) \neq\{0\}\}$ is a discrete set in $(X-*)$, called the points or support of $c$,

- $c(x) \perp c(y)$ for $x \neq y$.

The space of such configurations is denoted by $\operatorname{Conf}(X ; H)$ and carries the topology given by the subbasis sets

$$
\mathcal{U}(W, L):=\left\{c \in \operatorname{Conf}(X ; H) \mid \bigoplus_{x \in W} c(x) \in L, \sigma(c) \cap \partial W=\varnothing\right\}
$$

for all pairs of open subsets $W \subset(X-*)$ and $L \subset \mathcal{G r}(H)$, where $W \subset K \subset(X-*)$ is contained in a compact set $K$.

This topology is quite intuitive: we can move configuration points with their attached coefficients around in $X$ and we can continuously change coefficients in the Grassmannian. When points meet, their coefficients add; when points run into the basepoint, they disappear from the configuration.

Remark 3.2 In [9] the authors give a more general definition for configuration spaces on the category of pairs of spaces. However, our spaces $\operatorname{Conf}(X ; H)$ correspond precisely to their configurations on the pair $(X, *)$ with coefficients in $H$. For general pairs $(X, A)$, they allow the support $\sigma(c)$ of a configuration $c$ to be nondiscrete on $A$, and there $c$ can also take infinite-dimensional subspaces of $H$ (or infinite-rank projection operators, respectively) as values. However, they require that the Hilbert space closure of the total sum of all coefficients of a configuration is $H$. Therefore our 
definition is equivalent on the pointed category: the coefficient of the basepoint $*$ would necessarily be the orthogonal complement of the sum of coefficients on $(X-*)$ which is redundant information. We call the algebraic direct sum $\mathcal{D}(c):=\bigoplus_{x \in(X-*)} c(x)$ the domain of a configuration $c \in \operatorname{Conf}(X ; H)$.

The basepoint $c_{\infty}$ of $\operatorname{Conf}(X ; H)$ is the trivial configuration with no points. A configuration $c \in \operatorname{Conf}(X ; H)$ can be written as a formal sum $\Sigma_{x \in \sigma(c)} x c(x)$. Note however that the index set is not necessarily finite, ie the configuration points can accumulate at $*$. Finite configurations form a subspace

$$
\widehat{\operatorname{Conf}}(X ; H):=\{c \in \operatorname{Conf}(X ; H) \mid \sigma(c) \text { finite }\} .
$$

Conf $(-; H)$ and $\operatorname{Conf}(-; H)$ are covariant functors from the category of pointed compact topological spaces with basepoint-preserving maps $\varphi: X \rightarrow Y$ to the category of pointed topological spaces and continuous maps

$$
\varphi_{*}: \operatorname{Conf}(X ; H) \rightarrow \operatorname{Conf}(Y ; H), \quad c \mapsto \varphi_{*}(c): y \mapsto \bigoplus_{x \in \varphi^{-1}(y)} c(x) .
$$

One can furthermore show that a basepoint-preserving homotopy on $X$ induces a continuous homotopy on the space of configurations on $X$. In particular, we have

$$
\operatorname{Conf}(X ; H) \sim \operatorname{Conf}(X ; H) .
$$

To see this choose a compact neighbourhood $K$ of the basepoint $*$ which retracts to $*$. Then this retraction defines a continuous homotopy on configuration spaces and the resulting configurations will have finitely many points since the only possible accumulation point was the basepoint $*$.

The spaces Cônf $(X ; H)$ can be filtered by the dimension of the domain of the configurations. More precisely, let $\widehat{\operatorname{Conf}}(X ; H)_{\operatorname{dim} \leqslant m}$ be the subspace of configurations $c \in \operatorname{Conf}(X ; H)$ with $\operatorname{dim}(\mathcal{D}(c)) \leqslant m$. Then

$$
\operatorname{colim}_{m \rightarrow \infty} \operatorname{Conf}(X ; H)_{\operatorname{dim} \leqslant m} \hookrightarrow \operatorname{Conf}(X ; H)
$$

is a weak homotopy equivalence (compare the argument with the analogous one in Hohnhold et al [9] for $\operatorname{Conf}_{n}$ ). This filtration is typically used to apply a criterion for quasifibrations by Dold and Thom [5]. With this one obtains for example that cofibrations in the category of pointed manifolds $A \rightarrow X \rightarrow X / A$ for $A$ closed and $*_{X} \in A$, induce quasifibrations in $\widehat{C o n f}(-; H)$; see Bödigheimer [3].

Remark 3.3 Finite configurations of points with coefficients in a space $X$ have been defined and studied in more general context, in particular in terms of $\Gamma$-spaces (see 
for example Segal [19; 18], Bödigheimer [3] and McDuff [15; 14]). Set

$$
\mathcal{C}(X ; H)^{(m)}:=\left(\coprod_{k \leqslant m} X^{k} \times \Sigma_{k} C_{k}(H)\right) / \sim
$$

where $C_{k}(H)$ consists of $k$-tuples of pairwise orthogonal elements of $\mathcal{G} r(H), \Sigma_{k}$ is the symmetric group acting by permutation of factors and the equivalence relation identifies elements

$$
\begin{aligned}
\left(x_{1}, \ldots, x_{k} ;\left(H_{1}, \ldots, H_{k}\right)\right) & \sim\left(\ldots, \hat{x}_{i}, \ldots, x_{j}, \ldots ;\left(\ldots, \hat{H}_{i}, \ldots, H_{i} \oplus H_{j}, \ldots\right)\right), \\
(\ldots, *, \ldots ; \ldots, H, \ldots) & \sim(\ldots, \widehat{*}, \ldots ; \ldots, \hat{H}, \ldots)
\end{aligned}
$$

where here $\widehat{(\cdot)}$ means removing $(\cdot)$ from the tuple. Then the inclusion

$$
\operatorname{colim}_{m \rightarrow \infty} \mathcal{C}(X ; H)^{(m)} \hookrightarrow \operatorname{Conf}(X ; H)
$$

is a homotopy equivalence (compare Hohnhold et al [9]). This gives us another filtration of the spaces $\operatorname{Conf}(X ; H)$, namely by number of configuration points. Note that in general, $\Gamma$-spaces provide suitable coefficient spaces for the construction of configuration spaces of our type.

Next we define symmetric, Clifford-linear configurations. Symmetric here means $\mathbb{Z} / 2$-invariant (a good reference for equivariant configuration spaces is Hauschild [6]). Assume $H$ comes with a grading involution $\epsilon$ and $X$ comes with a $\mathbb{Z} / 2$-action $\alpha$, where $\alpha$ preserves the basepoint $*$ of $X$ and where $*$ again has a neighbourhood which $\mathbb{Z} / 2$-equivariantly retracts to $*$. Then

$$
\operatorname{Conf}^{\mathbb{Z} / 2}(X ; H):=\{c \in \operatorname{Conf}(X ; H) \mid \epsilon \circ c \circ \alpha=c\} .
$$

The adjective Clifford-linear concerns the coefficients: Let $H_{n}$ be an infinite-dimensional, $\mathbb{Z} / 2$-graded Hilbert space with compatible graded right $\mathcal{C}_{n}$-module structure. Let $\mathcal{G} r_{\mathcal{C}_{n}}\left(H_{n}\right)$ denote the Grassmannian of ungraded $\mathcal{C}_{n}$-submodules of $H_{n}$. Then a configuration $c \in \operatorname{Conf}\left(X ; H_{n}\right)$ is called $\mathcal{C}_{n}$-linear, if it factors through $\mathcal{G} r_{C_{n}}\left(H_{n}\right)$, ie if all coefficients of the configuration are $\mathcal{C}_{n}$-submodules of $H_{n}$. We call the space of such configurations $\operatorname{Conf}_{\mathcal{C}_{n}}\left(X ; H_{n}\right)$. Note that for a symmetric configuration $c \in \operatorname{Conf}_{\mathcal{C}_{n}}^{\mathbb{Z} / 2}\left(X ; H_{n}\right)$ the symmetric sums $c(x) \oplus c(\alpha(x))$ of coefficients will be graded $\mathcal{C}_{n}$-modules.

Remark 3.4 The results stated above for Conf $(-; H)$ hold true for $\operatorname{Conf}_{\mathcal{C}_{n}}^{\mathbb{Z} / 2}\left(-; H_{n}\right)$, as long as all maps are symmetry preserving (compare Markert [13, Theorem 2.2] and Hohnhold et al [9]): 
(i) For any fixed $H_{n}$ as above and $n \in \mathbb{Z}, \operatorname{Conf}_{\mathcal{C}_{n}}^{\mathbb{Z} / 2}\left(-; H_{n}\right)$ (and also $\widehat{C o n f}_{\mathcal{C}_{n}}^{\mathbb{Z} / 2}\left(-; H_{n}\right)$ ) is a functor from well-pointed compact Hausdorff $\mathbb{Z} / 2$-spaces with $\mathbb{Z} / 2$-equivariant maps to pointed topological spaces with continuous maps. We have again a homotopy equivalence

$$
\operatorname{Conf}_{\mathcal{C}_{n}}^{\mathbb{Z} / 2}\left(X ; H_{n}\right) \sim \operatorname{Conf}_{\mathcal{C}_{n}}^{\mathbb{Z} / 2}\left(X ; H_{n}\right)
$$

This is done as described above, contracting a $\mathbb{Z} / 2$-invariant compact neighbourhood $K$ of the basepoint $*$.

(ii) $\mathrm{Conf}_{\mathcal{C}_{n}}^{\mathbb{Z} / 2}\left(-; H_{n}\right)$ takes $\mathbb{Z} / 2$-equivariant cofibrations of manifolds to quasifibrations. The proof works completely analogously as in Bödigheimer [3], using the criterion of Dold and Thom where the filtration is given by the dimension of the domains. Note however that we are now counting dimensions in the sense of irreducible graded $\mathcal{C}_{n}$-modules.

(iii) The filtration properties for finite configurations still hold: the inclusions

and

$$
\operatorname{colim}_{m \rightarrow \infty} \operatorname{Conf}_{\mathcal{C}_{n}}^{\mathbb{Z} / 2}\left(X ; H_{n}\right)_{\operatorname{dim} \leqslant m} \hookrightarrow \operatorname{Conf}_{\mathcal{C}_{n}}^{\mathbb{Z} / 2}\left(X ; H_{n}\right)
$$

$$
\operatorname{colim}_{m \rightarrow \infty} \mathcal{C}_{\mathcal{C}_{n}}^{\mathbb{Z} / 2}\left(X ; H_{n}\right)^{(m)} \hookrightarrow \operatorname{Conf}_{\mathcal{C}_{n}}^{\mathbb{Z} / 2}\left(X ; H_{n}\right)
$$

are homotopy equivalences.

(iv) Concerning the second input slot, $\operatorname{Conf}_{\mathcal{C}_{n}}^{\mathbb{Z} / 2}(X ;-)$ is a homotopy functor (compare Bödigheimer [3]). Composition of configuration maps with a Clifford-linear isometric isomorphism of label spaces is an isomorphism of configuration spaces, independent of symmetry. In fact, this depends continuously on the choice of such isomorphism: For two label spaces $H_{n}, \widetilde{H}_{n}$, the map

$$
\psi: \operatorname{Conf}_{\mathcal{C}_{n}}\left(X ; H_{n}\right) \times \operatorname{ISO}_{\mathcal{C}_{n}}\left(H_{n}, \widetilde{H}_{n}\right) \rightarrow \operatorname{Conf}_{\mathcal{C}_{n}}\left(X ; \widetilde{H}_{n}\right), \quad(c, \beta) \mapsto \beta \circ c
$$

is continuous. Here $\operatorname{ISO}_{\mathcal{C}_{n}}\left(H_{n}, \widetilde{H}_{n}\right)$ stands for isometric isomorphisms from $H_{n}$ to $\widetilde{H}_{n}$.

Let now $\overline{\mathbb{R}}:=\mathbb{R} \cup\{\infty\}$ be the one-point compactification of $\mathbb{R}$ with basepoint $\{\infty\}$, equipped with the symmetry involution $\alpha: \overline{\mathbb{R}} \rightarrow \overline{\mathbb{R}}, \quad t \mapsto-t$. Recall the definition of the Hilbert universes $\mathcal{H}_{n}$. Then we set

$$
\operatorname{Conf}_{n}:=\operatorname{Conf}_{\mathcal{C}_{n}}^{\mathbb{Z} / 2}\left(\overline{\mathbb{R}} ; \mathcal{H}_{n}\right), \quad \operatorname{Conf}_{n}:=\operatorname{Conf}_{\mathcal{C}_{n}}^{\mathbb{Z} / 2}\left(\overline{\mathbb{R}} ; \mathcal{H}_{n}\right)
$$

It is shown in [9], that these spaces have the homotopy type of CW-complexes and can be filtered using a filtration of the Hilbert universe by finite-dimensional submodules $\cdots \hookrightarrow \mathcal{H}_{n}(i) \hookrightarrow \mathcal{H}_{n}(i+1) \hookrightarrow \cdots$. That is, we have a homotopy equivalence

$$
\operatorname{colim}_{i \rightarrow \infty} \operatorname{Conf}_{n}\left(\mathcal{H}_{n}(i)\right) \hookrightarrow \operatorname{Conf}_{n} .
$$


Moreover these spaces have the special property that we can associate to a configuration an operator on the Hilbert space whose spectral data is encoded in the configuration. In general configuration spaces with suitable input variables ( $H$ a Hilbert space, $X=\overline{\mathbb{R}}, \overline{\mathbb{C}}, \ldots$ ) correspond in this way to certain classes of operators; however the spaces $\operatorname{Conf}_{n}$ represent precisely the generators of super semigroup homomorphisms in $\mathcal{S G O}_{n}$ (see below, Lemma 3.7). Let us make this correspondence precise.

\subsection{Generating operators}

A configuration $c \in \operatorname{Conf}_{n}$ can be thought of as describing an operator $C$ with eigenvalues in $\mathbb{R}$, defined on $\mathcal{D}(c) \subset \mathcal{H}_{n}$, simply by taking the formal sum $\sum x \pi_{c(x)}$ to be the spectral decomposition of $C$, ie the eigenvalues of $C$ are the points of the configurations, the eigenspaces are the coefficients. We sometimes call $C$ the infinitesimal generator of the configuration $c$. Since the configurations might have points accumulating at $\pm \infty$, this might describe unbounded operators; however, thanks to their discrete spectrum, these operators are still quite well-behaved.

We denote by $\operatorname{Inf}_{n}$ the set of odd self-adjoint operators $C$ on a closed subspace $\overline{\mathcal{D}(C)} \subset \mathcal{H}_{n}$ which have compact resolvent and are $\mathcal{C}_{n}$-linear. The subspace $\overline{\mathcal{D}(C)}$ is part of the data, even though we do suppress its notation in the elements of $\operatorname{Inf}_{n}$. Note that a self-adjoint operator is in general only densely defined; in our case this means that $C \in \operatorname{Inf}_{n}$ is densely defined on $\overline{\mathcal{D}(C)}$. By the spectral theorem, an operator $C \in \operatorname{Inf}_{n}$ has discrete real eigenvalues which can accumulate at $\pm \infty$, and pairwise orthogonal finite-dimensional eigenspaces. We denote by $\operatorname{Esp}(C ; \lambda)$ the eigenspace of $C$ at $\lambda$, and by $\sigma(C)$ the spectrum of $C$ (the double use of $\sigma$ may be excused since it is consistent in our setting). Note that the grading on $\mathcal{H}_{n}$ induces a grading on the spaces of operators. Odd operators will have eigenvalue distributions symmetric with respect to $\alpha: \mathbb{R} \mapsto \mathbb{R}, t \mapsto-t$. So we have:

Lemma 3.5 For every $n$, there is a bijection $\operatorname{Conf}_{n} \leftrightarrow \operatorname{Inf}_{n}$.

This is immediate by sending $C \in \operatorname{Inf}_{n}$ to the configuration $c: \lambda \mapsto \operatorname{Esp}(C ; \lambda)$. Obviously, $c$ is of the correct type, with $\overline{\mathcal{D}(c)}=\overline{\mathcal{D}(C)}$ and $\sigma(C)=\sigma(c)$. Conversely, a configuration $c \in \operatorname{Conf}_{n}$ maps to $C:=\sum_{\lambda} \lambda \pi_{c(\lambda)}$. This is an odd operator since we started with a grading preserving configuration. Clearly the two maps are inverse to each other.

We can topologize the spaces $\operatorname{Inf}_{n}$ by making the above bijection a homeomorphism. Equivalently (see Markert [13]), define a norm topology on compact supports, ie the coarsest topology which makes the functional calculus

$$
\vartheta_{f}: \operatorname{Inf}_{n} \rightarrow \mathcal{B}_{\mathcal{C}_{n}}\left(\mathcal{H}_{n}\right), \quad C \mapsto f(C)
$$


continuous with respect to the norm topology on $\mathcal{B}_{\mathcal{C}_{n}}\left(\mathcal{H}_{n}\right)$, for all compactly supported continuous functions

$$
f \in C_{c}(\mathbb{R}):=\left\{f \in C_{0}(\mathbb{R}) \mid \operatorname{supp}(f) \subset \mathbb{R} \text { compact }\right\} .
$$

The proof that this is equivalent is technically a bit involved, however intuitively quite clear: the topology on Conf $_{n}$ controls sums of labels on bounded open subsets $W$ of $\mathbb{R}$, ie away from the basepoint. Functional calculus of an operator with a suitable characteristic function of such an open set $W$ produces precisely the orthogonal projection onto the sum of eigenspaces with eigenvalues in $W$. One can compare neighbourhood bases of the two topologies to see that they are equivalent.

Remark 3.6 (i) Obviously the homeomorphisms above restrict to homeomorphisms on the subspaces of finite configurations and finite generators, respectively, ie

$$
\text { Conf }_{n} \approx \operatorname{Inf}_{n}:=\left\{G \in \operatorname{Inf}_{n} \mid \operatorname{dim}(\mathcal{D}(G))<\infty\right\} .
$$

(ii) Functional calculus with basepoint-preserving, $\mathbb{Z} / 2$-equivariant continuous functions $f: \overline{\mathbb{R}} \rightarrow \overline{\mathbb{R}}$ induces continuous maps $f_{*}: \operatorname{Inf}_{n} \rightarrow \operatorname{Inf}_{n}$ (the same holds of course for $\left.\operatorname{Inf}_{n}\right)$. This follows directly since compositions $s \circ f$ of $f$ with an $s \in C_{c}(\mathbb{R})$ are again in $C_{c}(\mathbb{R})$. In particular, we also have homotopy equivalences $\operatorname{Inf}_{n} \sim \operatorname{Inf}_{n}$ for each $n$, given by applying functional calculus with a homotopy as in Remark 3.4(i) to the operators. Such a homotopy would push eigenvalues with absolute value larger than some positive number out to $\pm \infty$.

(iii) The spaces $\operatorname{Inf}_{n}$ filter by dimension of domain; that is, the inclusions

$$
\operatorname{colim}_{m \rightarrow \infty}\left(\operatorname{Inf}_{n}\right)_{\operatorname{dim} \leqslant m} \hookrightarrow \operatorname{Inff}_{n}
$$

are homotopy equivalences [9], where $\left(\operatorname{Inf}_{n}\right)_{\operatorname{dim} \leqslant m}:=\left\{G \in \operatorname{Inf}_{n} \mid \operatorname{dim}_{\mathcal{C}_{n}} \mathcal{D}(G) \leqslant m\right\} \approx$ $\left(\mathrm{Conf}_{n}\right)_{\text {dim } \leqslant m}$. Of course we also obtain, as in Remark 3.4, a filtration by the maximal number of (pairs of) eigenvalues, ie a homotopy equivalence

$$
\operatorname{colim}_{m \rightarrow \infty} \mathcal{C}_{n}^{(m)} \rightarrow \operatorname{Inf}_{n}
$$

where $\mathcal{C}_{n}^{(m)}:=\mathcal{C}_{\mathcal{C}_{n}}^{\mathbb{Z} / 2}\left(\overline{\mathbb{R}} ; \mathcal{H}_{n}\right)^{(m)}$. Again the spaces have the homotopy type of CWcomplexes, and we have a homotopy equivalence

$$
\operatorname{colim}_{i \rightarrow \infty} \operatorname{Inf}_{n}\left(\mathcal{H}_{n}(i)\right) \hookrightarrow \operatorname{Inf}_{n} .
$$

(iv) Remark 3.4(iv) can also be restated in terms of generating operators: let $\operatorname{Inf}_{n}(H)$ denote the operators of type Inf (ie odd, compact resolvent, self-adjoint on closure of domain and Clifford-linear) on a Hilbert space $H$ with compatible $\mathcal{C}_{n}$-structure. Then 
the conjugation of operators in $\operatorname{Inf}_{n}(H)$ with a Clifford-linear isomorphism $\beta: H \rightarrow \tilde{H}$ is a homeomorphism and depends continuously on the isomorphism:

$$
\psi: \operatorname{Inf}_{n}(H) \times \operatorname{ISO}_{\mathcal{C}_{n}}(H, \tilde{H}) \rightarrow \operatorname{Inf}_{n}(\tilde{H}), \quad(C, \beta) \mapsto \beta \circ C \circ \beta^{-1}
$$

is continuous with fiber $\operatorname{ISO}_{\mathcal{C}_{n}}(H, \tilde{H})$.

(v) The notation both of $\operatorname{Inf}_{n}$ and $\operatorname{Conf}_{n}$ suppresses the fact that the objects are odd or symmetric, respectively. It would be more standard to define the operator class $\operatorname{Inf}_{n}$ without the condition that the operators be odd, and then write $\operatorname{Inf}_{n}^{\text {odd }}$ and $\operatorname{Inf}_{n}^{\mathrm{ev}}$. We avoid the notation in the odd case, since this is almost exclusively what we use and we keep the notation for the even part wherever we need it. Certainly, for every $n$ one has again

$$
\operatorname{Conf}_{n}^{\mathrm{ev}} \approx \operatorname{Inf}_{n}^{\mathrm{ev}} \sim \operatorname{Inf}_{n}^{\mathrm{ev}}
$$

where $\operatorname{Conf}_{n}^{\text {ev }}$ consists of $\mathbb{Z} / 2$-equivariant configurations but now with respect to the trivial symmetry involution $\alpha^{\mathrm{ev}}:=\mathrm{id}_{\overline{\mathbb{R}}}$ and the grading involution $\epsilon$ (in particular all coefficients are graded $\mathcal{C}_{n}$-modules).

(vi) We think of the configurations as a geometric —or combinatorial—model for operators in $\operatorname{Inf}_{n}$ : it provides an intuitive idea of the topology on the spaces $\operatorname{Inf}_{n}$. In the following chapter containing the proofs of our statements we will use the operator language since it is best to calculate with, but we will supply pictures of the configurations where it seems helpful.

Now the following is an easy consequence of Theorem 2.2:

Lemma 3.7 For every $n$ there are homeomorphisms $\mathcal{S G O}_{n} \approx \operatorname{Inf}_{n}$ and homotopy equivalences:

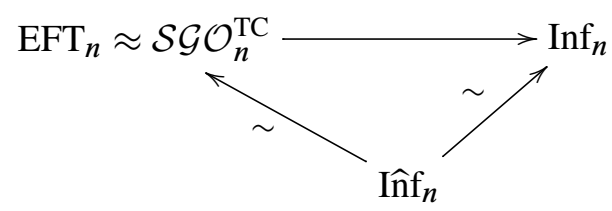

Proof By Theorem 2.2, every element $E \in \mathcal{S G O}_{n}$ has a uniquely determined infinitesimal generator $G_{E}$. The properties of the spectrum of this generator follow from the properties of the image operators $e^{-t G_{E}^{2}+\theta G_{E}}=e^{-t G_{E}^{2}}\left(1+\theta G_{E}\right)$ of the corresponding generalized super semigroup. In particular, the family of operators $e^{-t G_{E}^{2}}$ for $t \in \mathbb{R}_{>0}$ has to lie in $\mathcal{K}_{\mathcal{C}_{n}}\left(\mathcal{H}_{n}\right)^{\text {ev }}$. This in turn implies that $G_{E}$ has compact resolvent, is selfadjoint on the closure of its domain and of course $\mathcal{C}_{n}$-linear. In addition $G_{E}$ has to be odd since $e^{-t G_{E}^{2}} G_{E} \in \mathcal{K}_{\mathcal{C}_{n}}\left(\mathcal{H}_{n}\right)^{\text {odd }}$. Thus $G_{E}$ is an element of $\operatorname{Inf}_{n}$. This produces 
a set bijection between $\mathcal{S G O}{ }_{n}$ and $\operatorname{Inf}_{n}$. Furthermore, if $E \in \mathrm{EFT}_{n} \approx \mathcal{S G O}_{n}^{\mathrm{TC}}$ was the generator of a field theory, then the only property distinguishing $G_{E}$ from an operator in $\operatorname{Inf}_{n}$ is the fact that $e^{-t G_{E}^{2}}$ has to be trace class for $t \in \mathbb{R}_{>0}$, ie that the eigenvalues of $G_{E}$ have to converge "fast" to infinity. Thus the bijection takes the subspace $\mathcal{S G O}_{n}^{\mathrm{TC}} \subset \mathcal{S G O} \mathcal{O}_{n}$ to the subspace $\operatorname{Inf}_{n}^{\mathrm{TC}} \subset \operatorname{Inf}_{n}$ which consists of operators $G$ such that $e^{-t G^{2}}$ is trace-class for $t \in \mathbb{R}_{>0}$. The bijection is even a homeomorphism. Now it is easy to check that a homotopy equivalence as in Remark 3.6(ii) above retracts $\mathrm{Inf}_{n}^{\mathrm{TC}}$ to the smaller subspace of finite-domain operators Inff ${ }_{n}$ (see Hohnhold et al [9]; compare Markert [13, Lemma 57] and Hohnhold [8]). This also proves the claims in Remark 2.5(i).

Remark 3.8 Of course one can produce spaces $\mathcal{S} \widehat{\mathcal{G}} \mathcal{O}_{n} \approx \operatorname{Inf}_{n}$ by setting $\mathcal{S} \widehat{\mathcal{G}} \mathcal{O}_{n}:=$ $\mathcal{S G O}\left(\mathcal{F R}_{\mathcal{C}_{n}}\left(\mathcal{H}_{n}\right)\right)$, where the latter consists of self-adjoint $\mathcal{C}_{n}$-linear finite-rank operators. Clearly then we have $\mathcal{S} \widehat{\mathcal{G}} \mathcal{O}_{n} \stackrel{\sim}{\hookrightarrow} \mathcal{S G O} \mathcal{G}_{n}$.

In Hohnhold et al [9] the spaces $\operatorname{Inf}_{n}$ are also related directly to various other models of $\mathrm{KO}$, among these in particular the Atiyah-Singer $\Omega$-spectrum of Fredholm operators. The original spaces $\mathcal{F}_{n}$ of Atiyah and Singer consist of skew-adjoint $\mathcal{C}_{n-1}$-antilinear Fredholm operators on a Hilbert universe $H_{n}$ with $\mathcal{C}_{n-1}$ structure, satisfying an extra condition in dimensions $n=3 \bmod 4$. These can equivalently be described as spaces $\mathcal{F}_{n}^{\mathrm{gr}}$ of odd self-adjoint Fredholm operators on a $\mathbb{Z} / 2$-graded $\mathcal{C}_{n}$-Hilbert universe, which are $\mathcal{C}_{n}$-linear (and also satisfy a corresponding condition in the mentioned cases). The authors give homotopy equivalences

$$
\Phi_{n}: \mathcal{F}_{n}^{\mathrm{gr}} \stackrel{\sim}{\rightarrow} \operatorname{Inf}_{n}
$$

for positive $n$ (depending on the model of the Hilbert universes, their proof works also for negative $n$ ). The map $\Phi_{n}$ breaks into two parts: the first one maps into graded $\mathcal{C}_{n}-$ linear configurations on $\overline{\overline{\mathbb{R}}}:=\mathbb{R} \cup\{ \pm \infty\}$, $\operatorname{Conf}_{\mathcal{C}_{n}}^{\mathbb{Z} / 2}\left(\overline{\overline{\mathbb{R}}},\{ \pm \infty\} ; \mathcal{H}_{n}\right.$ ) (compare Remark 3.2). The second part is the obvious map

$$
\operatorname{Conf}_{\mathcal{C}_{n}}^{\mathbb{Z} / 2}\left(\overline{\bar{R}},\{ \pm \infty\} ; \mathcal{H}_{n}\right) \rightarrow \operatorname{Conf}_{n} \cong \operatorname{Conf}_{\mathcal{C}_{n}}^{\mathbb{Z} / 2}\left(\overline{\mathbb{R}},\{\infty\} ; \mathcal{H}_{n}\right)
$$

which is a quasifibration with contractible fibers. Recall here that the spectrum of a Fredholm operator $T$ consists of two parts, the essential spectrum, and the discrete spectrum, $\sigma(T)=\sigma_{\text {discrete }}(T) \cup \sigma_{\text {ess }}(T)$, where the first one comes from the operator $\pi(T)$ obtained from $T$ under the quotient map to the Calkin algebra $\pi: \mathcal{B}\left(\mathcal{H}_{n}\right) \rightarrow$ $\mathcal{B}\left(\mathcal{H}_{n}\right) / \mathcal{K}\left(\mathcal{H}_{n}\right)$. Since a Fredholm operator is invertible up to compact operators, this essential part of the spectrum has a gap around 0 with the gap width $\epsilon(T)$ depending continuously on $T$. For odd self-adjoint Fredholm operators $T \in \mathcal{F}_{n}^{\mathrm{gr}}, \sigma(T)$ is contained in $\mathbb{R}$, symmetric about 0 , and bounded. That is, the spectral data of $T$ is not 
a configuration in our usual sense: the essential spectrum can be continuous, therefore we do not necessarily have a discrete configuration of points on $\mathbb{R}$ (however, within the gap of $\sigma_{\text {ess }}(T)$, we do). Note also that the domain of $T$ is all of $\mathcal{H}_{n}$. However, due to the gap in the essential spectrum we do have a configuration of the above type up to homotopy. The first map above is simply given by functional calculus with a homotopy contracting everything outside the gap of $\sigma_{\text {ess }}(T)$ to the two points $\{ \pm \infty\}$. Thus the information contained in a Fredholm operator $T$ is reduced to a part of its spectrum, namely the eigenvalues of the compact part of $T$ which lie within the gap of the essential spectrum of $T$.

We can write this as follows.

$$
\Phi_{n}: \mathcal{F}_{n}^{\mathrm{gr}} \rightarrow \operatorname{Inf}_{n}, \quad T \mapsto \sum_{\left|\lambda_{i}\right|<\epsilon(T)} \frac{\lambda_{i} \epsilon(T)}{\epsilon(T)^{2}-\lambda_{i}^{2}} \pi_{\operatorname{Esp}\left(T ; \lambda_{i}\right)}
$$

This is particularly useful for describing the spectrum map for the spaces $\operatorname{Inf}_{n}$. We have a description of it for Fredholm operators (for $n \geqslant 0$ ) given by Atiyah-Singer (see for example Lawson and Michelson [12, Chapter III.10]) as

$$
b: \mathcal{F}_{n+1}^{\mathrm{gr}} \rightarrow \Omega \mathcal{F}_{n}^{\mathrm{gr}}, \quad T \mapsto\left(t \mapsto b(T)_{t}:=\cos (t) \epsilon e_{n+1}+\sin (t) T, t \in[0, \pi]\right) .
$$

For our purposes we would like to rescale this using arccot: $\overline{\mathbb{R}} \rightarrow[0, \pi]$ to obtain

$$
b: \mathcal{F}_{n+1}^{\mathrm{gr}} \rightarrow \Omega \mathcal{F}_{n}^{\mathrm{gr}}, \quad T \mapsto\left(x \mapsto b(T)_{x}:=\frac{1}{\sqrt{1+x^{2}}}\left(-x \in e_{n+1}+T\right), x \in \overline{\overline{\mathbb{R}}}\right) .
$$

Now we set

$$
\tilde{b}: \operatorname{Inf}_{n+1} \rightarrow \Omega \operatorname{Inf}_{n}, \quad G \mapsto\left(x \mapsto b(G)_{x}:=-x \epsilon e_{n+1}+G, x \in \overline{\overline{\mathbb{R}}}\right) .
$$

It is easy to check that these operators are elements of $\operatorname{Inf}_{n}$; in particular, they do not commute with $e_{n+1}$ anymore. Note that the operators $b(G)_{x}$ have domain $\mathcal{D}(G)$ for $x \in \mathbb{R}$ and trivial domain for $x \in\{ \pm \infty\}$, ie the images $b(G)$ are loops at the basepoint of $\operatorname{Inf}_{n}$. We do not particularly denote this subtlety in the following.

Lemma 3.9 The map $\tilde{b}$ is a homotopy equivalence fitting in the homotopy commutative diagram:

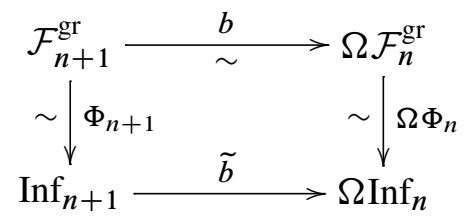


Proof We can simply calculate the loops of configurations-or operators respectivelyresulting from $\tilde{b}\left(\Phi_{n+1}(T)\right)$ and $\Omega \Phi_{n}(b(T))$ in terms of their points and labels. We obtain

$$
\begin{aligned}
\tilde{b}\left(\Phi_{n+1}(T)\right)_{x} & =\sum_{\lambda^{2}<\epsilon(T)^{2}} \frac{ \pm 1}{\epsilon(T)^{2}-\lambda^{2}} \sqrt{a(x, T, \lambda)} \pi_{V^{ \pm}(T)}, \\
\Omega \Phi_{n}(b(T))_{x} & =\sum_{\lambda^{2}<\epsilon(T)^{2}} \frac{ \pm 1}{\epsilon(T)^{2}-\lambda^{2}} \sqrt{b(x, T, \lambda)} \pi_{V^{ \pm}(T)},
\end{aligned}
$$

where $V^{ \pm}(T)$ are the eigenspaces of $\left(\epsilon e_{n+1}+T\right)$ at $\pm \sqrt{1+\lambda^{2}}$ and where

$$
\begin{aligned}
& a(x, T, \lambda)=x^{2} \epsilon(T)^{2}+\lambda^{2} \epsilon(T)^{2}-x^{2} \lambda^{2}, \\
& b(x, T, \lambda)=x^{2} \epsilon(T)^{2}+\lambda^{2} \epsilon(T)^{2}+x^{2} \lambda^{2}+x^{4} .
\end{aligned}
$$

This shows that there is a homotopy $H_{t}$ by functional calculus between the two maps above, depending continuously on $T, x$ and $t$.

Remark 3.10 For convenience in our presentation of the Bott map for Inf, we can apply the isomorphism inv: $\Omega \operatorname{Inf}_{n} \rightarrow \Omega \operatorname{Inf}_{n}: \gamma \mapsto \gamma^{-1}$. By abuse of notation we denote the resulting map again by $b$

$$
b: \operatorname{Inf}_{n+1} \rightarrow \Omega \operatorname{Inf}_{n}, \quad G \mapsto\left(x \mapsto b(G)_{x}=x \epsilon e_{n+1}+G, x \in \overline{\overline{\mathbb{R}}}\right) .
$$

Remark 3.11 The Bott map for Fredholm operators cited above is given in Lawson and Michelson [12] for positive $n$. A translation of it for negative values of $n$ can be found in Joachim [10]. This can analogously be rewritten for the Inf-model.

\subsection{Connective Covers}

Recall that we can interpret the right $\mathcal{C}_{n+k}$-modules $\mathcal{H}_{n+k}$ as $\mathcal{C}_{-k}-\mathcal{C}_{n}$-bimodule (denoted by $\mathcal{H}_{n}^{k}$ ) by replacing the last $k$ generators of $\mathcal{C}_{n+k}$ acting from the right by generators of $\mathcal{C}_{-k}$ acting from the left. Now let $\operatorname{Inf}_{n}\left(\mathcal{H}_{n}^{k}\right)$ denote $\mathcal{C}_{n}$-linear operators of type Inf defined on $\mathcal{H}_{n}^{k}$.

Definition 3.12 For $k \geqslant 0$, we set

$$
\operatorname{Inf}_{n}^{k}:=\left\{G \in \operatorname{Inf}_{n}\left(\mathcal{H}_{n}^{k}\right) \mid G^{2} \text { is } \mathcal{C}_{-k}-\mathcal{C}_{n} \text {-linear }\right\} .
$$

Analogously we define $\operatorname{Conf}_{n}^{k}$ as a subset of $\operatorname{Conf}_{\mathcal{C}_{n}}^{\mathbb{Z} / 2}\left(\mathbb{R} ; \mathcal{H}_{n}^{k}\right)$ by the condition that symmetric sums $g(x) \oplus g(-x)$ of coefficients of a configuration $g \in \operatorname{Conf}_{n}^{k}$ will 
be graded $\mathcal{C}_{-k}-\mathcal{C}_{n}$-bimodules. It is clear that $\operatorname{Conf}_{n}^{k} \approx \operatorname{Inf}_{n}^{k}$. Furthermore, $\operatorname{Inf}_{n}^{k} \sim$ $\operatorname{Inf} f_{n}^{k}:=\operatorname{Inf}_{n}^{k} \cap \operatorname{Inf}_{n}\left(\mathcal{H}_{n}^{k}\right)$ by the usual argument.

Similarly as for $\operatorname{Inf}_{n}$, the spaces $\operatorname{Inf}_{n}^{k}$ and $\operatorname{Inff}_{n}^{k}$ are also homotopy equivalent to the colimits of filtrations (compare Remark 3.6). We show here the more involved argument concerning filtration by finite subspaces of the Hilbert universe:

Lemma 3.13 The spaces $\operatorname{Inf}_{n}^{k}$ have the homotopy type of $\mathrm{CW}$-complexes. Moreover, there is a filtration by subcomplexes

$$
\ldots \rightarrow \operatorname{Inff}_{n}^{k}\left(\mathcal{H}_{i}\right) \rightarrow \operatorname{Inf}_{n}^{k}\left(\mathcal{H}_{i+1}\right) \rightarrow \ldots \operatorname{colim}_{i \rightarrow \infty} \operatorname{Inf}_{n}^{k}\left(\mathcal{H}_{i}\right) \stackrel{\sim}{\hookrightarrow} \operatorname{Inf}_{n}^{k}
$$

given by an increasing filtration of $\mathcal{H}_{n}^{k}$ by graded $\mathcal{C}_{n+k}$-submodules of $\mathcal{H}_{i}$ of Clifford dimension $i$, such that in particular $\mathcal{G} r_{C_{n+k}}\left(\mathcal{H}_{n}^{k}\right) \sim \operatorname{colim}_{i \rightarrow \infty} \mathcal{G} r_{\mathcal{C}_{n+k}}\left(\mathcal{H}_{i}\right)$. The maps in the colimit are the obvious ones induced by the inclusions of the $\mathcal{H}_{i}$.

Proof We show that all spaces concerned have the homotopy type of CW-complexes and that the inclusion

$$
\operatorname{colim}_{i \rightarrow \infty} \operatorname{In̂f}_{n}^{k}\left(\mathcal{H}_{i}\right) \hookrightarrow \operatorname{Inf}_{n}^{k}
$$

is a homotopy equivalence. Both statements result from the fact that all the above mentioned spaces are realizations of "good" simplicial spaces (in the sense of Segal [17]) which satisfy the requirements levelwise.

In [9] the authors show that the spaces $\operatorname{Inf}_{n}$ are homotopy equivalent to the classifying spaces of certain internal space categories $\mathfrak{C}_{n}$. We will use the same idea, modified for our purposes. Let $\mathfrak{C}_{n}^{k}$ be the category consisting of finite-dimensional graded $\mathcal{C}_{n+k}$-submodules of $\mathcal{H}_{n}^{k}$ as objects. This object set is topologized as the subspace $\operatorname{Proj}_{\mathcal{C}_{n+k}}\left(\mathcal{H}_{n}^{k}\right) \subset \mathcal{B}_{\mathcal{C}_{n+k}}\left(\mathcal{H}_{n}^{k}\right)$ of finite-rank, Clifford-linear, orthogonal projection operators (recall that orthogonal projection onto some $\mathcal{C}_{n+k}$-submodule is a $\mathcal{C}_{n+k}-$ linear operator). Morphisms between $W_{0}$ and $W_{1}$ exist if and only if $W_{0} \subseteq W_{1}$, and then they are defined as odd, orthogonal, $\mathcal{C}_{n}$-linear involutions on the complement $W_{1}-W_{0}$ of $W_{0}$ in $W_{1}$, that is

$$
\mathfrak{C}_{n}^{k}\left(W_{0}, W_{1}\right)=\left\{R \in \mathcal{O}\left(W_{1}-W_{0}\right) \mid R^{2}=\mathrm{Id}, R \text { odd, } R e_{i}=e_{i} R, i=1, \ldots, n\right\} .
$$

The morphisms can be understood as a subspace of $\mathcal{B}_{\mathcal{C}_{n}}\left(\mathcal{H}_{n}^{k}\right) \times \operatorname{Proj}_{\mathcal{C}_{n+k}}\left(\mathcal{H}_{n}^{k}\right)^{2}$. For simplicity we will denote this internal space category by $\mathfrak{C}$ in the following. Using the notation of Hohnhold et al [9], an $m$-simplex $x=\left(W_{i} ; R_{i}\right)$ in the nerve $\mathfrak{N C}$ is given by a chain of objects ( $W_{0} \subseteq W_{1} \subseteq \ldots \subseteq W_{m}$ ) and involutions $R_{i}$ as above on $W_{i}-W_{i-1}, i=1, \ldots, m$. Roughly, a point $(x, t)$ in the corresponding simplex of the geometric realization describes a finite configuration by making the submodules 
$W_{i}-W_{i-1}$ with their involutions correspond to pairs of labels sitting at pairs of points in $\mathbb{R}$ given by the coordinates $\pm t_{i}$ of $t \in \Delta^{m}$. The proof that

$$
B \mathfrak{C}=|\mathfrak{N C}| \sim \operatorname{Inf}_{n}^{k}
$$

works exactly as in [9]. Their category $\mathfrak{C}_{n}$ differs from ours only in the fact that the objects are $\mathcal{C}_{n}$-submodules instead of $\mathcal{C}_{n+k}$-submodules. They show that the $m$-skeleta $B \mathfrak{C}_{n}^{(m)}$ of the geometric realization are homeomorphic to $\mathcal{C}_{n}^{(m)}$ (compare again Remark 3.4). One can check that this goes through in our case (in each level $m$, $B \mathfrak{C}^{(m)}$ is homeomorphic to $\left.\left(\mathcal{C}_{n}^{k}\right)^{(m)}\right)$.

Furthermore, the argument goes through as well after replacing $\mathcal{H}_{n}^{k}$ by the finitedimensional submodule $\mathcal{H}_{i}$. Going through the proof in Hohnhold et al [9] we obtain subcomplexes $B \mathfrak{C}\left(\mathcal{H}_{i}\right)=\left|\mathfrak{N C}\left(\mathcal{H}_{i}\right)\right|$ of $B \mathfrak{C}$ and see that these are homotopy equivalent to the spaces $\operatorname{Inf}_{n}^{k}\left(\mathcal{H}_{i}\right)$.

To see that these spaces have the homotopy type of CW-complexes, we can look at the structure of the simplicial space $\mathfrak{N C}$ (or $\mathfrak{N C}\left(\mathcal{H}_{i}\right)$, respectively): it suffices to see that this is a simplicial object in the category of spaces of the homotopy type of CW-spaces and that it is good in the sense of Segal [17]. This will imply (see Segal [19, Proposition A.1]) that its geometric realization has the same property.

First we note that each level space $\mathfrak{N \mathfrak { C } _ { m }}$ is the total space of a fiber bundle: let

$$
\begin{aligned}
\operatorname{Flag}_{m}:=\mathcal{F l a g}_{m}\left(\mathcal{H}_{n}^{k}\right): & =\left\{\left(P_{0}, \ldots, P_{m}\right) \in\left(\operatorname{Proj}_{\mathcal{C}_{n+k}}\left(\mathcal{H}_{n}^{k}\right)\right)^{m+1} \mid P_{i} P_{i+k}=P_{i}\right\} \\
& \approx\left\{\left(W_{0}, \ldots, W_{m}\right) \in\left(\mathcal{G r}_{\mathcal{C}_{n+k}}\left(\mathcal{H}_{n}^{k}\right)\right)^{m+1} \mid W_{i} \subseteq W_{i+1}\right\}
\end{aligned}
$$

Note here that $\mathcal{F l a g}_{m}$ is a disjoint union of components given by the dimension vectors of length $m$

$$
\mathcal{F l a g}_{m}=\coprod_{d=\left(d_{0} \leqslant d_{1} \leqslant \ldots \leqslant d_{n}\right) \in \mathbf{N}^{m+1}} \mathcal{F l a g}{ }_{m}^{d}
$$

where $m$ is the signatures of the flags and where the dimension $d_{i}$ denotes the Clifforddimension $\operatorname{dim}_{\mathcal{C}_{n+k}}\left(W_{i}\right)$. Denote by $P_{W_{i}-W_{i-1}}$ the orthogonal projections onto the submodules $W_{i}-W_{i-1}$. Then we can write

$$
\begin{aligned}
\mathfrak{N C} \mathfrak{C}_{m}=\left\{\left(W_{0}, \ldots, W_{m} ; A\right) \in\right. & \text { Flag }_{m} \times \mathcal{O}_{\mathcal{C}_{n}}^{\text {odd }}\left(W_{m}-W_{0}\right) \\
& \left.\mid A^{2}=\mathrm{Id}, A P_{W_{i}-W_{i-1}}=P_{W_{i}-W_{i-1}} A, i=1, \ldots, m\right\}
\end{aligned}
$$

where we have replaced the $m$ involutions $R_{i}$ on the subsequent complements in the flag by one operator $A$ on $W_{m}-W_{0}$ which preserves the subspaces $W_{i}-W_{i-1}$ and defines involutions there. Then we get a map $\mathfrak{N C}_{m} \rightarrow \mathcal{F}$ lag $_{m}$ taking an element in $\mathfrak{N C}$ to its flag. 
This is a subbundle of the Hom-bundle of the tautological bundle over $\mathcal{F} l a g_{m}$. The tautological bundle $E_{m}$ over it is clearly a fiber bundle over each component $\mathcal{F} l a g_{m}^{d}$ whose fiber is a flag of finite-dimensional graded $\mathcal{C}_{n+k}$-modules (as vector spaces the fibers are isomorphic to $\left(\mathbb{R}^{d_{0} \mathfrak{d}_{n+k}}, \ldots, \mathbb{R}^{d_{m} \mathfrak{d}_{n+k}}\right)$, where $\mathfrak{d}_{n+k}$ is the real dimension of an irreducible $\mathcal{C}_{n+k}$-module). Its transition functions are maps into $\mathcal{C}_{n+k}$-linear orthogonal operators which preserve the subspace structure. It is then easy to check that $\mathfrak{N \mathfrak { C } _ { m }}$ forms a fiber bundle over each component, where the fiber is the space of odd, $\mathcal{C}_{n}$-linear involutions on the flags (ie on $W_{m}-W_{0}$, keeping the subspaces $W_{i}-W_{i-1}$ invariant). The spaces $\mathcal{F} \operatorname{lag}_{m}$ have the homotopy type of CW-complexes, and so do the fibers: therefore also the total spaces $\mathfrak{N C}_{m}$.

Furthermore, the simplicial space $\mathfrak{N C}$ is good, ie the inclusions of the degenerated subspaces

$$
s_{i}\left(\mathfrak{N C}_{m-1}\right)=: \mathfrak{N C}_{m, i} \hookrightarrow \mathfrak{N C}_{m}
$$

are strict neighbourhood retracts in $\mathfrak{N C}_{m}$. This can be easily seen by connectivity arguments: the inclusion maps $\mathfrak{N C}_{m, i}$ surjectively onto those connected components of $\mathfrak{N \mathfrak { C } _ { m }}$ with dimension vectors of the form $\left(d_{0}, \ldots, d_{i}, d_{i}, \ldots, d_{m-1}\right)$.

Goodness of the simplicial space implies that the fat realization and the realization are homotopy equivalent; the fat realization preserves homotopy type of CW-complexes (see Segal [19]). Therefore the spaces have the homotopy type of CW-complexes.

Moreover, we obtain a homotopy equivalence

$$
\left|\operatorname{colim}_{i \rightarrow \infty} \mathfrak{N C}\left(\mathcal{H}_{i}\right)\right| \rightarrow|\mathfrak{N C}|
$$

by levelwise considerations. In each level $m$ the inclusion of Hilbert spaces induces a homotopy equivalence

$$
\operatorname{colim}_{i \rightarrow \infty} \mathcal{F} \operatorname{lag}_{m}\left(\mathcal{H}_{i}\right) \stackrel{\sim}{\hookrightarrow} \mathcal{F} \operatorname{lag}_{m}
$$

by classical arguments. ${ }^{1}$ In fact, we have pullback diagrams:

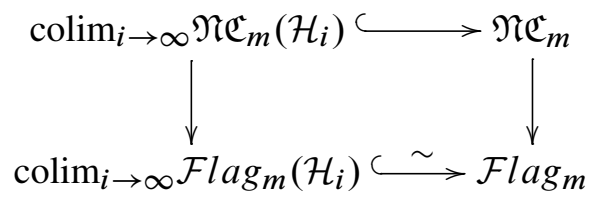

Therefore the inclusion of the total spaces is a homotopy equivalence as well. Since both $\mathfrak{N C}$ and $\operatorname{colim}_{i \rightarrow \infty} \mathfrak{N C}\left(\mathcal{H}_{i}\right)$ are good, this induces a homotopy equivalence as

\footnotetext{
${ }^{1}$ In fact one can use a similar strategy as above and show the homotopy equivalence on the level of the total spaces of GL-frame bundles over $\mathcal{F} l a g_{m}^{d}$; this homotopy then descends to the flag spaces. The idea is in Milnor and Stasheff's book [16].
} 
wanted, again by the quoted result of Segal. Finally, since realization commutes with colim $_{\rightarrow \infty}$, we see that also

$$
\operatorname{colim}_{i \rightarrow \infty}\left|\mathfrak{N C}\left(\mathcal{H}_{i}\right)\right| \stackrel{\sim}{\rightarrow}|\mathfrak{N C}|
$$

is a homotopy equivalence.

In the next section we will determine the homotopy type of the spaces $\operatorname{Inf}_{n}^{k}$. In some sense, this easy first observation inspired the definition of $\operatorname{Inf}_{n}^{k}$ :

Proposition 3.14 The spaces $\operatorname{Inf}_{n}^{k}$ are connected for $k \geqslant 1$.

Proof Let $G \in \operatorname{Inf}_{n}^{k}$ where $k \geqslant 1$, and regard the first additional generator $f_{1}$ of the algebra $\mathcal{C}_{-k}$ acting by left multiplication (here we use our standard names as defined before). This generator $f_{1}$ squares to +1 and commutes with $G^{2}$ as well as with all $n$ generators $e_{1}, \ldots, e_{n}$, of $\mathcal{C}_{n}$. It acts as an odd operator on $\operatorname{ker}(G)=\operatorname{ker}\left(G^{2}\right)$, with eigenvalues $( \pm 1)$. The path

$$
H_{t}(G):=\left(\frac{1}{1-t}\right) G+\left(\frac{t}{1-t}\right) \pi_{\operatorname{ker}(G)} f_{1}, \quad t \in[0,1],
$$

splits the kernel (ie the middle label of the configuration of $G$ ) and then moves all eigenvalues symmetrically out to infinity, where they disappear. One checks that for all $t, H_{t}(G)$ is in $\operatorname{Inf}_{n}^{k}$, and that the summands commute with each other. In particular $H_{t}(G)^{2}$ is $\mathcal{C}_{-k}-\mathcal{C}_{n}$-linear since $G^{2}$ is (and therefore also $\left.\left(\pi_{\operatorname{ker}(G)} f_{1}\right)^{2}=\pi_{\mathrm{ker}\left(G^{2}\right)}\right)$. In terms of configurations, this path does not change the symmetric sums of coefficients (the eigenspaces of $G^{2}$ ), only their distribution on the real axis. The operators $H_{t}(G)$ have eigenvalues

$$
\lambda_{t}= \begin{cases} \pm\left(\frac{t}{1-t}\right) & \text { on } \operatorname{ker}(G), \\ \left(\frac{1}{1-t}\right) \lambda & \text { on } \operatorname{ker}(G)^{\perp},\end{cases}
$$

where the $\lambda$ are the eigenvalues of $G$. Hence this gives a path from $G$ to the basepoint in $\operatorname{Inf}_{n}^{k}$.

Remark 3.15 This argument does not imply that $\operatorname{Inf}_{n}^{k}, k \geqslant 1$, is contractible. The paths connecting points $G$ in the space with the basepoint do not depend continuously on $G$. For instance (thinking in terms of configurations) one could easily imagine a continuous path of configurations along which a symmetric pair of configuration points runs into 0 where the two coefficient spaces are added; however, the homotopy we chose above might split this label into two different parts. 


\section{Proofs}

\subsection{Outline}

The goal of this section is to prove the following.

Theorem 4.1 For $k \geqslant 0$ and all $n$, there are weak homotopy equivalences

$$
\operatorname{Inf}_{n}^{k} \stackrel{\sim}{\longrightarrow} \Omega \operatorname{Inf}_{n-1}^{k+1}, \quad G \mapsto\left(x \mapsto x f_{n}+G, x \in \overline{\overline{\mathbb{R}}}\right) .
$$

Together with Proposition 3.14 this implies that $\operatorname{Inf}_{n}^{k}$ is $(k-1)$-connected, since for $i \leqslant k-1$, we have

$$
\pi_{i} \operatorname{Inf}_{n}^{k} \cong \pi_{0} \Omega^{i} \operatorname{Inf}_{n}^{k} \cong \pi_{0} \operatorname{Inf}_{n+i}^{k-i} \cong 0 .
$$

Note that for $i=k$ we obtain that $\pi_{k} \operatorname{Inf}_{n}^{k} \cong \pi_{0} \operatorname{Inf}_{n+k}^{0} \cong \pi_{0} \operatorname{Inf}_{n+k}$ (by definition $\operatorname{Inf}_{n}^{0}$ is equal to $\operatorname{Inf}_{n}$ ). Moreover, we will show that the maps $c_{k}: \operatorname{Inf}_{n}^{k} \rightarrow \operatorname{Inf}_{n}$ given by simply forgetting the extra structure (ie conjugating the operators in $\operatorname{Inf}_{n}^{k}$ with a $\mathcal{C}_{n}$-linear isomorphism between $\mathcal{H}_{n}^{k}$ and $\mathcal{H}_{n}$ ), induce isomorphisms under $\pi_{i}$ for $i \geqslant k$. That is, $\Omega^{k} c_{k}$ are weak homotopy equivalences. To see this we show:

Lemma 4.2 The map $c_{1}$ fits into a diagram:

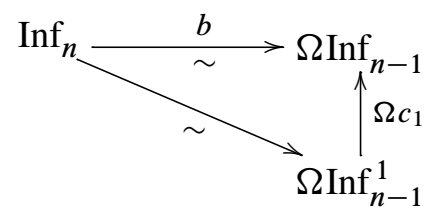

Proof Simply note that the operators $b(G)_{x}$ satisfy the condition for $\operatorname{Inf}_{n-1}^{1}$, namely: $b(G)_{x}^{2}=x^{2} \mathrm{Id}+G^{2}$ commutes with all $e_{i}, i=1, \ldots, n-1$ and with $f_{n}$ (while $b(G)_{x}$ itself does not commute with neither $e_{n}$ nor $f_{n}$ ).

Summarizing, we see that the $c_{k}$ are higher covers and thus we obtain:

Corollary 4.3 For $k \geqslant 0$, the spaces $\operatorname{Inf}_{n}^{k}$ are $(k-1)$-connected covers of the spaces $\operatorname{Inf}_{n}$,

$$
\operatorname{Inf}_{n}^{k} \sim \operatorname{Inf}_{n}\langle k\rangle
$$

Using Lemma 3.7 which compares the spaces $\mathcal{S G O}$ and Inf we can reformulate this in terms of $\mathcal{S G O}$ and obtain Theorem 2.3 and thus also Theorem 2.4. 


\subsection{Construction of quasifibrations}

We will show that for $k \geqslant 0$ and all $n$, there are quasifibrations with contractible total space:

$$
\mathrm{Inf}_{n}^{k} \rightarrow \widehat{\mathcal{T}}_{n}^{k} \stackrel{p}{\rightarrow} \mathrm{Inff}_{n-1}^{k+1}
$$

Using the inclusion of the fiber together with a suitable contraction of the total space, we will obtain a map $b_{p}$ : $\operatorname{Inf}_{n}^{k} \stackrel{\sim}{\rightarrow} \Omega \operatorname{Inf}_{n-1}^{k+1}: G \mapsto\left(t \mapsto \ln (t) f_{n}+G, t \in[0, \infty]\right)$ : this is clearly equivalent to $b$ up to rescaling and homotopy. To define the total spaces, let $\mathrm{Inf}_{n, k}^{\text {ev,pos }}$ denote even, $\mathcal{C}_{-k}-\mathcal{C}_{n}$-linear operators of type Inf on $\mathcal{H}_{n}^{k}$ which have positive spectrum, ie eigenvalues in $[0, \infty)$. The operators $E \in \operatorname{Inf}_{n, k}^{\text {ev,pos }}$ generate finite, nonsymmetric configurations on $\overline{\mathbb{R}}_{\geqslant 0}$ whose coefficients are graded $\mathcal{C}_{-k}-\mathcal{C}_{n}-$ submodules of $\mathcal{H}_{n}^{k}$. We want to pair operators of this type with operators of type $\operatorname{Inf}_{n}^{k}$, to obtain a space configurations on the pointed cone $\overline{\mathbb{R}} \wedge \overline{\mathbb{R}}_{\geqslant 0}=\left(\mathbb{R} \times \mathbb{R}_{\geqslant 0}\right) \cup\{\infty\}$, where the symmetry involution is given by

$$
\bar{\alpha}: \overline{\mathbb{R}} \wedge \overline{\mathbb{R}}_{\geqslant 0} \rightarrow \overline{\mathbb{R}} \wedge \overline{\mathbb{R}}_{\geqslant 0}, \quad(\lambda, \mu) \mapsto(-\lambda, \mu) .
$$

These will be our total spaces. To be precise, we set

$$
\widehat{\mathcal{T}}_{n}^{k}:=\operatorname{Conf}_{\mathcal{C}_{n}}^{\mathbb{Z} / 2}\left(\overline{\mathbb{R}} \wedge \overline{\mathbb{R}}_{\geqslant 0} ; \mathcal{H}_{n}^{k}\right)
$$

where the symmetry involutions are given by $\bar{\alpha}$ and the grading involution $\epsilon$ on $\mathcal{H}_{n}^{k}$. In terms of operators we can describe such a configuration as a triple $(\mathcal{D} ; G, E)$ where $\mathcal{D} \subset \mathcal{H}_{n}^{k}$ is a graded, finite-dimensional, $\mathcal{C}_{-k}-\mathcal{C}_{n}$-linear closed subspace (the domain of the configuration), $G$ is an operator in $\mathrm{Inf}_{n}^{k}$ and $E$ an operator in $\mathrm{Inf}_{n, k}^{\text {ev,pos }}$, such that $\mathcal{D}(G)=\mathcal{D}(E)=\mathcal{D}$ and $[G, E]=0$ on $\mathcal{D}$. There is a set bijection between the set of such triples and configurations as above: A pair of commuting operators $(G, E)$ with common domain $\mathcal{D}$ generates a symmetric configuration on the cone $\overline{\mathbb{R}} \wedge \overline{\mathbb{R}} \geqslant 0$. Simply note that one can apply the spectral theorem to both and obtain a decomposition of $\mathcal{D}$ into simultaneous eigenspaces. Then let

$$
c_{(\mathcal{D} ; G, E)}: \mathbb{R} \times \mathbb{R}_{\geqslant 0} \rightarrow \mathcal{G} r_{\mathcal{C}_{n}}\left(\mathcal{H}_{n}^{k}\right), \quad(\lambda, \mu) \mapsto \operatorname{Esp}(E ; \mu) \cap \operatorname{Esp}(G ; \lambda) \subset \mathcal{D} .
$$

This produces a configuration in $\widehat{\mathcal{T}}_{n}^{k}$ (the $\mathbb{Z} / 2$-invariance of $c_{(\mathcal{D} ; G, E)}$ follows easily from the fact that $G$ is odd and $E$ even). Vice versa a configuration $c$ determines a pair of operators by setting $E:=\sum_{\lambda, \mu} \mu \pi_{c(\lambda, \mu)}$ and $G:=\sum_{\lambda, \mu} \lambda \pi_{c(\lambda, \mu)}$, with common domain $\mathcal{D}(G)=\mathcal{D}(E)=\mathcal{D}(c)$.

For convenience we will use the operator description of $\widehat{\mathcal{T}}_{n}^{k}$ and most of the times suppress the notation of the domain as well.

Lemma 4.4 The spaces $\widehat{\mathcal{T}}_{n}^{k}$ are contractible. 
Proof Functional calculus with a contracting homotopy like

$$
\underline{H}: \overline{\mathbb{R}}_{\geqslant 0} \times I \rightarrow \overline{\mathbb{R}}_{\geqslant 0}, \quad(\mu, t) \mapsto\left((1-t) \mu+\frac{t}{1-t}\right)
$$

induces a continuous contraction of $\operatorname{Inf}_{n, k}^{\mathrm{ev}, \mathrm{pos}}$ by $H_{t} E:=\underline{H}_{t}(E)$ (compare Remark 3.6(ii)). We obtain a well-defined continuous homotopy on $\widehat{\mathcal{T}}_{n}^{k}$ by setting $H_{t}(G, E):=$ $\left(G, H_{t} E\right)$. Note that even though there is a jump in domains this is continuous as a map of configurations. In Figure 1 we see how one can contract the configurations on $\overline{\mathbb{R}} \wedge \overline{\mathbb{R}}_{\geqslant 0}$ by moving the points along a vertical flow to the basepoint, without changing the labels.

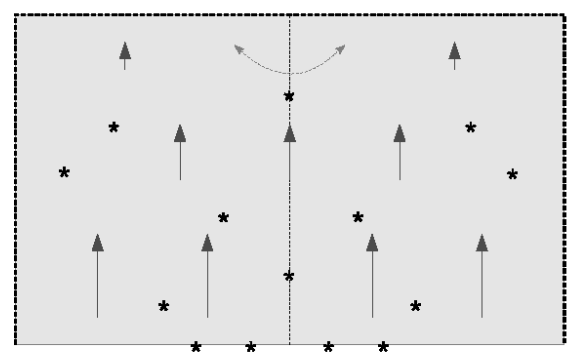

Figure 1: A contracting homotopy on $\widehat{\mathcal{T}}_{n}^{k}$. The picture shows configurations on $\mathbb{R} \times[0, \infty)$; the dashed boundary lines should be thought as collapsed to obtain the cone $\overline{\mathbb{R}} \wedge \overline{\mathbb{R}}_{\geqslant 0}$. Configuration points are indicated as stars and the symmetry axis is indicated by a dotted line. Labels at the points are not shown.

For the definition of the map $p$, recall the notation concerning generators of the Clifford action on $\mathcal{H}_{n+k}$ (we use $e_{i}$ for a generator acting from the right, and $f_{i}:=\epsilon e_{i}$ for the corresponding generator acting from the left). We think of the operator pairs in $\widehat{\mathcal{T}}_{n}^{k}$ as being compatible with the structure of $\mathcal{H}_{n}^{k}$, while the operators in the image $\operatorname{Inf}_{n-1}^{k+1}$ should be compatible with the structure of $\mathcal{H}_{n-1}^{k+1}$. Now set

$$
p: \widehat{\mathcal{T}}_{n}^{k} \rightarrow \operatorname{Inff}_{n-1}^{k+1}, \quad(\mathcal{D} ; G, E) \mapsto G+\ln (E) f_{n}=: P_{G, E}
$$

where the domain of $P_{G, E}$ is defined as $\mathcal{D}\left(P_{G, E}\right):=\mathcal{D} \cap \operatorname{ker}(E)^{\perp}$. One checks that the map $p$ is well-defined: for $i=1, \ldots, n-1, P_{G, E}$ commutes with $e_{i}$ since the $e_{i}$ anticommute both with $e_{n}$ and $\epsilon$ and commute by definition with $G$ and $E$. However $P_{G, E}$ does not commute with $e_{n}$, since $e_{n}$ anticommutes with $\ln (E) f_{n}$ but commutes with $G$. Therefore $P_{G, E}$ is $\mathcal{C}_{n-1}$-linear but not $\mathcal{C}_{n}$-linear with respect to the given generators, whereas $P_{G, E}^{2}=G^{2}+(\ln (E))^{2}$ is $\mathcal{C}_{-(k+1)}-\mathcal{C}_{n-1}$-bilinear: It commutes 
with the $f_{n+1}, \ldots, f_{n+k}$ by definition and with $f_{n}=\epsilon e_{n}$ as well since both parts commute.

Furthermore, $P_{G, E}^{2}$ has the eigenvalues $r^{2}:=\left(\lambda^{2}+(\ln \mu)^{2}\right)$ and eigenspaces

$$
\operatorname{Esp}\left(P_{G, E}^{2} ; r^{2}\right)=\bigoplus_{\lambda^{2}+(\ln \mu)^{2}=r^{2}} \operatorname{Esp}(G ; \lambda) \cap \operatorname{Esp}(E ; \mu)
$$

where $\pm \lambda, \mu$ are eigenvalues of $G$ and $E$, respectively. Thus $P_{G, E}$ itself has eigenvalues $\pm r= \pm \sqrt{\lambda^{2}+(\ln \mu)^{2}}$. In particular, the spectrum of $P_{G, E}$ is discrete and finite.

One can show that $p$ is continuous. We have seen that the eigenvalues of the operators in the image, $P_{G, E}=G+\ln (E) f_{n}$ depend continuously on the eigenvalues of $G$ and $E$. Furthermore, the eigenspaces of $P_{G, E}^{2}$ depend continuously on the eigenspaces of $G$ and $E$. What is left to see is that the root $P_{G, E}$ has eigenspaces which depend continuously on $G$ and $E$. This requires some technical effort. We skip the precise argument here, as it involves mainly detailed exercises with the topology of the spaces (see Markert [13, Section 3.2.2]). To give an intuitive argument, consider what $p$ does in terms of configurations: as illustrated in Figure 2, $p$ collects all configuration points on a curve $r^{2}=\lambda^{2}+(\ln \mu)^{2}$, adds up all coefficients, and splits this sum using the involution $f_{n}$ to produce two new labels which will be distributed at $\pm r$. This process depends continuously on the configurations on the cone.

Next let us analyze the preimage of an operator $P$ in the image of $p$. Recall that $\mathcal{D}(P) \oplus \operatorname{ker}(E)=\mathcal{D}(G)=\mathcal{D}(E)$, for $(G, E)$ in the fiber $p^{-1}(P)$. We can recover certain parts of the data of $(G, E)$ by comparing $P$ with $f_{n}$. Calculating the graded commutator of $P$ and $f_{n}$ on $\mathcal{D}(P)$ returns $\ln (E)$ and thus also $\left.G\right|_{\mathcal{D}(P)}$. However, the subspace $\operatorname{ker}(E)$ and its decomposition into eigenspaces of $G$ cannot be recovered. We obtain a bijection

$$
\operatorname{Inf}_{n}^{k}\left(\mathcal{D}(P)^{\perp}\right) \longleftrightarrow p^{-1}(P)
$$

where $\operatorname{Inf}_{n}^{k}\left(\mathcal{D}(P)^{\perp}\right)$ is the subspace of $\operatorname{Inf}_{n}^{k}$ consisting of operators $T$ with $\mathcal{D}(T) \subset$ $\mathcal{D}(P)^{\perp}$. The bijection sends and operator $T \in \operatorname{Inf}_{n}^{k}\left(\mathcal{D}(P)^{\perp}\right)$ to the pair of operators $\left(E_{P}(T), G_{P}(T)\right)$ defined on $\mathcal{D}(T) \oplus \mathcal{D}(P)$, where both operators depend only on $P$ on the part $\mathcal{D}(P)$ of their domain and only on $T$ on $\mathcal{D}(T)$ :

$$
\begin{array}{lll}
\left.E_{P}(T)\right|_{\mathcal{D}(P)}:=\exp \left(\frac{1}{2}\left[P, f_{n}\right]\right) & & \left.G_{P}(T)\right|_{\mathcal{D}(P)}:=P-\frac{1}{2}\left[P, f_{n}\right] f_{n} \\
\left.E_{P}(T)\right|_{\mathcal{D}(T)}:=0 & & \left.G_{P}(T)\right|_{\mathcal{D}(T)}:=T
\end{array}
$$

This is a homeomorphism; its inverse is given by sending a pair $(G, E)$ in the preimage of $P$ to $\left.G\right|_{\operatorname{ker}(E)}=\left.G\right|_{\mathcal{D}(P)^{\perp}}$. Clearly this depends continuously on $G$ and $E$. It follows immediately that $p$ is surjective. In particular the preimage of the basepoint 


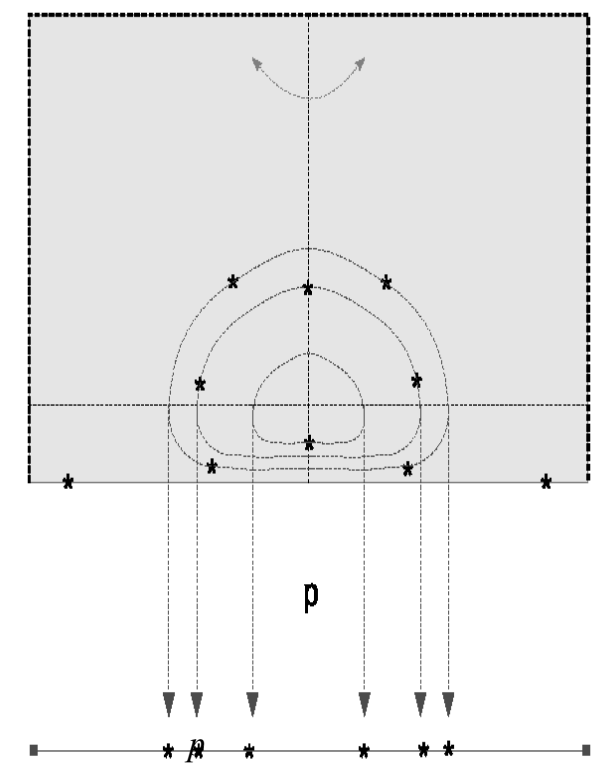

Figure 2: The map $p$ on configuration spaces. The indicated curves sketch the lines $r^{2}=\lambda^{2}+(\ln \mu)^{2}$.

in $\mathrm{Inf}_{n-1}^{k+1}$ is homeomorphic to $\mathrm{Inf}_{n}^{k}$. In terms of configurations, the inclusion of this fiber is induced by the inclusion of the line $\mathbb{R} \times\{0\} \hookrightarrow \mathbb{R} \times \mathbb{R} \geqslant 0$. Next we study the homotopy type of general fibers.

Proposition 4.5 An inclusion $i: H \hookrightarrow H \oplus V$ of infinite-dimensional graded $\mathcal{C}_{-k}-$ $\mathcal{C}_{n}$-bimodules with finite codimension induces a weak homotopy equivalence

$$
i_{*}: \operatorname{Inf}_{n}^{k}(H) \stackrel{\sim}{\longrightarrow} \operatorname{Inf}_{n}^{k}(H \oplus V) .
$$

Corollary 4.6 Let $P, S \in \mathrm{Inf}_{n-1}^{k+1}$, with $\mathcal{D}(P) \subset \mathcal{D}(S)$. Then there is a weak homotopy equivalence of the fibers

$$
p^{-1}(S) \stackrel{\sim}{\longrightarrow} p^{-1}(P)
$$

induced by the inclusion $\mathcal{D}(S)^{\perp} \hookrightarrow \mathcal{D}(P)^{\perp}$.

The corollary is immediate, since the domains of all operators $P, S$ in $\operatorname{Inf}_{n-1}^{k+1}$ differ only by some finite-dimensional subspace $V$, ie

$$
p^{-1}(P) \approx \operatorname{Inf}_{n}^{k}\left(\mathcal{D}(P)^{\perp}\right), \quad p^{-1}(S) \approx \operatorname{Inf}_{n}^{k}\left(\mathcal{D}(P)^{\perp} \oplus V\right) .
$$


Proof of Proposition 4.5 Roughly, we argue that there will be "enough room" to deform domains which hit the finite-dimensional subspace $V$ into ones which lie outside $V$. As infinite-dimensional Clifford-modules the two label spaces $H$ and $H \oplus V$ are isomorphic. Choosing such an isomorphism $\psi$ gives maps

$$
\mathrm{Inf}_{n}^{k}(H) \underset{\psi_{*}}{\stackrel{i_{*}}{\gtrless}} \mathrm{Inf}_{n}^{k}(H \oplus V) .
$$

We now want to show the weak homotopy equivalence by checking that for compact CW-complexes $K$, the induced map

$$
\left[K, \operatorname{Inf}_{n}^{k}(H)\right] \stackrel{i_{*}}{\longrightarrow}\left[K, \operatorname{Inf}_{n}^{k}(H \oplus V)\right]
$$

is an isomorphism. Now note that-as a consequence of Lemma 3.13-each continuous map $c: K \rightarrow \operatorname{Inff}_{n}^{k}$ for $K$ compact and $C W$, factors up to homotopy through $\operatorname{Inf}_{n}^{k}\left(H_{m}\right)$ for some $m$. That is, for each such map $c$ we can always find a finite-dimensional subspace $H_{c}$ of $H$ such that there is a homotopy commutative diagram:

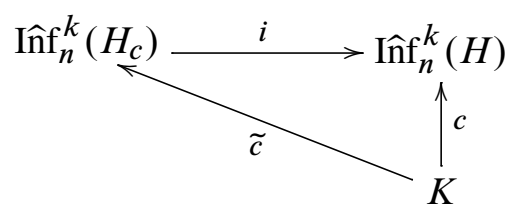

This implies that a map $c \in\left[K, \operatorname{Inf}_{n}^{k}(H)\right]$ is homotopic to its factorization

$$
K \stackrel{\tilde{c}}{\longrightarrow} \operatorname{Inf}_{n}^{k}\left(H_{c}\right) \stackrel{j_{*}}{\longrightarrow} \operatorname{Inf}_{n}^{k}(H) .
$$

This combination is itself homotopic to its composition with

$$
\operatorname{Inf}_{n}^{k}(H) \stackrel{i_{*}}{\longrightarrow} \operatorname{Inf}_{n}^{k}(H \oplus V) \stackrel{\psi_{*}}{\longrightarrow} \operatorname{Inf}_{n}^{k}(H) .
$$

The maps $j$ and $\psi \circ i \circ j$ are homotopic since both are infinite-codimension embeddings in $\operatorname{Emb}\left(H_{c} ; H\right) \sim *$. Therefore they induce homotopic maps $j_{*} \sim \psi_{*} \circ i_{*} \circ j_{*}$.

\subsection{Quasifibration properties}

In the following we show that for $n, k \geqslant 0$, the maps $p: \widehat{\mathcal{T}}_{n}^{k} \rightarrow \mathrm{Inf}_{n-1}^{k+1}$ are quasifibrations with fiber $\mathrm{Inf}_{n}^{k}$. A surjective map $q: X \rightarrow Y$ is a quasifibration, if for all $y \in Y$ and all $x \in q^{-1}(y)$ one has $\pi_{i}\left(X, q^{-1}(y) ; x\right) \cong \pi_{i}(Y ; y)$ for all $i \geqslant 0$. The crucial point is that a quasifibration $q$ induces a long exact sequence in homotopy. To prove that a map $q$ is a quasifibration, one can use the well-known theorem of Dold and Thom [5]: 
Theorem 4.7 (Dold-Thom) Let $q: X \rightarrow Y$ be surjective. Then $q$ is a quasifibration, if there is an increasing filtration $\left\{F_{i} Y\right\}$ of $Y$, where $\operatorname{colim}_{i \rightarrow \infty} F_{i} Y \sim Y$ is a homotopy equivalence, such that the following holds:

- For every open subset $U$ of $B_{i}:=F_{i}-F_{i-1}$,

$$
p^{-1}(U) \rightarrow U
$$

is a fibration.

- For every $i$, there exist neighbourhoods $N_{i} \subset F_{i+1}$ of $F_{i}$ and a contracting homotopy $h: N_{i} \times I \rightarrow N_{i}$ with $h_{0}=\mathrm{id}_{N_{i}}$ and $h_{1}\left(N_{i}\right) \subset F_{i}$.

- This deformation $h$ is covered by a homotopy $H: q^{-1}\left(N_{i}\right) \times I \rightarrow q^{-1}\left(N_{i}\right)$, $H_{0}=\mathrm{id}_{q^{-1}\left(N_{i}\right)}$, such that for each point $x \in N_{i}$,

$$
H_{1}: q^{-1}(x) \rightarrow q^{-1}\left(h_{1}(x)\right)
$$

is a weak homotopy equivalence.

We define the filtration on $\operatorname{Inf}_{n-1}^{k+1}$ by

$$
F_{i}:=\left\{P \in \mathrm{Inff}_{n-1}^{k+1} \mid \operatorname{dim}_{\mathcal{C}_{n+k}} \mathcal{D}(P) \leqslant i\right\} .
$$

Here the dimension counts irreducible graded $\mathcal{C}_{n+k}$-modules (or respectively, $\mathcal{C}_{-k-1}-$ $\mathcal{C}_{n-1}$-bimodules). Using the same argument as for Remark 3.6(iii), one can see that the colimit over these spaces is homotopy equivalent to $\mathrm{Inf}_{n-1}^{k+1}$. Note that the inclusion of the colimit is a set-bijection; it is not a homeomorphism since the colimit space has more open sets based on open sets in $\overline{\mathbb{R}}$ around the basepoint. This can be helped by a homotopy equivalence contracting a neighbourhood of the basepoint. All these arguments have nothing to do with the additional Clifford-structure and all homotopy equivalences can be made through the correct spaces. This clearly gives a filtration with $F_{i} \subset F_{i+1}$, covering $\mathrm{Inf}_{n-1}^{k+1}$.

Proposition 4.8 The map $p$ is a fiber bundle on

$$
B_{i}=\left\{P \in \operatorname{Inff}_{n-1}^{k+1} \mid \operatorname{dim}_{\mathcal{C}_{n+k}} \mathcal{D}(P)=i\right\} .
$$

Proof We need to see that the map $p^{-1}\left(B_{i}\right) \rightarrow B_{i}$ has local trivializations. We first look at the domains of operators in $p^{-1}\left(B_{i}\right)$. Let

$$
\mathcal{D o m}_{i}^{\perp}:=\left\{(P ; v) \in B_{i} \times \mathcal{H}_{n}^{k} \mid v \in \mathcal{D}(P)^{\perp}\right\}
$$

and define the map $\quad \rho_{i}: \mathcal{D}_{i} \rightarrow B_{i}, \quad(P ; v) \mapsto P$. 
Obviously $\rho_{i}$ is a pullback of the complementary tautological bundle over the Grassmannian $\mathcal{G} r_{i}\left(\mathcal{H}_{n}^{k}\right)$

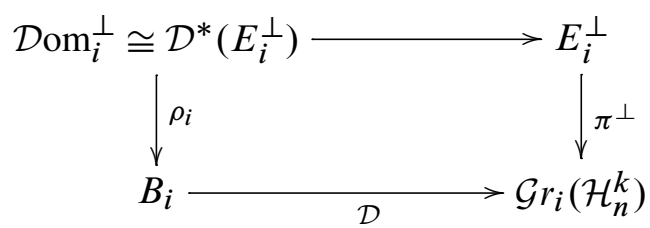

where $\mathcal{D}$ maps $P$ to its domain $\mathcal{D}(P) . E_{i}^{\perp}$ is a fiber bundle by the usual argument (extension of locally orthogonal sections; this works also in the Clifford-linear context). Therefore $\rho_{i}$ is a fiber bundle for all $i$. But if $\mathcal{D o m}_{i}^{\perp} \rightarrow B_{i}$ is locally trivial, then so is $p^{-1}\left(B_{i}\right) \rightarrow B_{i}$ since we can construct local trivializations by conjugating the operators in $p^{-1}\left(B_{i}\right)$ with the isomorphisms of the Hilbert spaces given by the local trivializations of $\mathcal{D o m}_{i}^{\perp} \rightarrow B_{i}$ (compare Remark 3.6(iv)).

Next we need to find neighbourhoods $N_{i} \subset F_{i+1} \subset \operatorname{Inf}_{n-1}^{k+1}$ of $F_{i}$ for every $i$, and contracting homotopies. There is a standard way to do this for configuration spaces, and we just rephrase it in terms of operators (to be consistent). We define neighbourhoods $N_{i} \subset F_{i+1}$ of $F_{i}$ by

$$
N_{i}:=\left\{P \in F_{i+1} \mid \operatorname{dim}_{\mathcal{C}_{n+k}}\left(\bigoplus_{y \in(-1,1)} \operatorname{Esp}(P ; y)\right) \leqslant i\right\} .
$$

This contains $F_{i}$ and also those operators in $F_{i+1}$ who have eigenvalues in $(-\infty,-1] \cup$ $[1,+\infty)=: U_{1}$. Now we contract the neighbourhood $U_{1} \cup\{\infty\}$ of the basepoint in $\overline{\mathbb{R}}$. This induces a continuous homotopy on $\mathrm{Inf}_{n-1}^{k+1}$ (compare Remark 3.6(ii)), and in particular we obtain a homotopy as wished

$$
h: N_{i} \times I \rightarrow N_{i}
$$

such that $h_{1}\left(N_{i}\right) \subset F_{i}$ and $h_{0}=\operatorname{id}_{N_{i}}$.

Lemma 4.9 This deformation $h$ is covered by a homotopy

$$
H: p^{-1}\left(N_{i}\right) \times I \rightarrow p^{-1}\left(N_{i}\right), \quad H_{0}=\mathrm{id}_{p^{-1}\left(N_{i}\right)}
$$

such that for each point $x \in N_{i}$,

$$
H_{1}: p^{-1}(x) \rightarrow p^{-1}\left(h_{1}(x)\right)
$$

is a weak homotopy equivalence.

It is clear how to cover this deformation in the total space of our map $p$. We contract the neighbourhood of the basepoint in $\overline{\mathbb{R}} \wedge \overline{\mathbb{R}}_{\geqslant 0}$ given by $(\lambda, \mu)$ with $\sqrt{\lambda^{2}+\ln (\mu)^{2}} \geqslant 1$, 
by a homotopy $\underline{H}$. In terms of configurations it is easy to see that we obtain a homotopy on $\widehat{\mathcal{T}}_{n}^{k}$. In terms of operators, we have to apply functional calculus with $\underline{H}_{t}$, only since we have pairs of operators we need to do this carefully. For pairs of commuting operators $(G, E) \in \widehat{\mathcal{T}}_{n}^{k}$, we can write out their simultaneous spectral decompositions, for instance

Then we set

$$
\begin{aligned}
G & =\sum_{(\lambda, \mu)} \operatorname{pr}_{1}(\lambda, \mu) \pi_{\operatorname{Esp}(G ; \lambda) \cap \operatorname{Esp}(E ; \mu) .} . \\
H_{t} G & :=\sum_{(\lambda, \mu)} \operatorname{pr}_{1}\left(\underline{H}_{t}(\lambda, \mu)\right) \pi_{\operatorname{Esp}(G ; \lambda) \cap \operatorname{Esp}(E ; \mu)}
\end{aligned}
$$

and analogously for $E$. This produces a covering homotopy on the preimages

$$
H: p^{-1}\left(N_{i}\right) \times I \rightarrow p^{-1}\left(N_{i}\right) .
$$

Recall that elements of the fiber over an operator $P$ are determined by operators $T \in \operatorname{Inf}_{n}^{k}\left(\mathcal{D}(P)^{\perp}\right)$. Thus as long as the domain of $h_{t} P$ does not change, ie for all $t \in[0,1)$, we have

$$
p^{-1}(P) \approx \operatorname{Inf}_{n}^{k}\left(\mathcal{D}(P)^{\perp}\right)=\operatorname{Inff}_{n}^{k}\left(\mathcal{D}\left(h_{t} P\right)^{\perp}\right) \approx p^{-1}\left(h_{t} P\right)
$$

and so

$$
p^{-1}\left(h_{t} P\right) \sim H_{t}\left(p^{-1}(P)\right)
$$

for $t \neq 1$. When $t=1, \mathcal{D}\left(h_{1} P\right)^{\perp}$ jumps by the sum of all eigenspaces of $P$ with eigenvalues in $U_{1}$. Thus for all $P \in N_{i}$ the map $H_{1}: p^{-1}(P) \rightarrow p^{-1}\left(h_{1} P\right)$ is given by inclusion of the fibers

$$
\operatorname{Inf}_{n}^{k}\left(\mathcal{D}(P)^{\perp}\right) \hookrightarrow \operatorname{Inf}_{n}^{k}\left(\mathcal{D}\left(h_{1} P\right)^{\perp}\right)=\operatorname{Inf}_{n}^{k}\left(\mathcal{D}(P)^{\perp} \oplus \bigoplus_{\lambda \in U_{1}} \operatorname{Esp}(P ; \lambda)\right) .
$$

But this is a weak homotopy equivalence by Proposition 4.5. Hence we proved the last condition of Dold-Thom.

Finally with this it is clear that there is a weak homotopy equivalence as required:

$$
\operatorname{Inff}_{n}^{k} \stackrel{\sim}{\longrightarrow} \Omega\left(\mathrm{Inff}_{n-1}^{k+1}\right)
$$

For this one compares a quasifibration with contractible total space $F \rightarrow E \stackrel{q}{\rightarrow} B$ like the above with the path-loop fibration of $B$. There is a commutative diagram

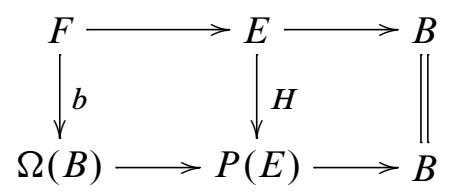

where $H$ is induced by a homotopy contracting the total space $E$ (this gives a path to the basepoint for every point of $E$, ie an element in the path space $P(E)$ ). Now both 
the quasifibration and the fibration induce long exact sequences in homotopy, and since both total spaces are contractible, the map $b$ induces an isomorphism in homotopy by the five-lemma and is thus a weak homotopy equivalence $F \stackrel{\sim}{\longrightarrow} \Omega(B)$. This completes the proof of Theorem 4.1.

\section{References}

[1] MF Atiyah, I M Singer, Index theory for skew-adjoint Fredholm operators, Inst. Hautes Études Sci. Publ. Math. (1969) 5-26 MR0285033

[2] MJ Behrens, A new proof of the Bott periodicity theorem, Topology Appl. 119 (2002) 167-183 MR1886093

[3] C-F Bödigheimer, Stable splittings of mapping spaces, from: "Algebraic topology (Seattle, Wash., 1985)", (HC Miller, D C Ravenel, editors), Lecture Notes in Math. 1286, Springer, Berlin (1987) 174-187 MR922926

[4] P Deligne, J W Morgan, Notes on supersymmetry (following Joseph Bernstein), from: "Quantum fields and strings: a course for mathematicians, Vol. 1, 2 (Princeton, NJ, 1996/1997)", (P Deligne, P Etingof, D S Freed, L C Jeffrey, D Kazhdan, J W Morgan, D R Morrison, E Witten, editors), Amer. Math. Soc. (1999) 41-97 MR1701597

[5] A Dold, R Thom, Quasifaserungen und unendliche symmetrische Produkte, Ann. of Math. (2) 67 (1958) 239-281 MR0097062

[6] H Hauschild, Äquivariante Konfigurationsräume und Abbildungsräume, from: "Topology Symposium (Proc. Sympos., Univ. Siegen, 1979)”, (U Koschorke, W D Neumann, editors), Lecture Notes in Math. 788, Springer, Berlin (1980) 281-315 MR585664

[7] N Higson, E Guentner, Group $C^{*}$-algebras and $K$-theory, from: "Noncommutative geometry", (S Doplicher, R Longo, editors), Lecture Notes in Math. 1831, Springer, Berlin (2004) 137-251 MR2058474

[8] H Hohnhold, Supersymmetry in the Stolz-Teichner project on elliptic cohomology, $\mathrm{PhD}$ thesis, University of California, San Diego (2006)

[9] H Hohnhold, S Stoltz, P Teichner, K-Theory: From minimal geodesics to super symmetric field theories. In memory of Raoul Bott, Preprint (2009) Available at http://math. berkeley.edu/ teichner/papers.html

[10] M Joachim, A symmetric ring spectrum representing KO-theory, Topology 40 (2001) 299-308 MR1808222

[11] M Joachim, $K$-homology of $C^{*}$-categories and symmetric spectra representing $K$ homology, Math. Ann. 327 (2003) 641-670 MR2023312

[12] H B Lawson, Jr, M-L Michelsohn, Spin geometry, Princeton Math. Series 38, Princeton Univ. Press (1989) MR1031992 
[13] E Markert, Connective 1-dimensional Euclidean field theories, $\mathrm{PhD}$ thesis, University of Notre Dame (2005) Available at http://etd.nd.edu/ETD-db/theses/ available/etd-07122005-151903/

[14] D McDuff, Configuration spaces of positive and negative particles, Topology 14 (1975) 91-107 MR0358766

[15] D McDuff, Configuration spaces, from: “ $K$-theory and operator algebras (Proc. Conf., Univ. Georgia, Athens, Ga., 1975)”, (B B Morrel, I M Singer, editors), Lecture Notes in Math. 575, Springer, Berlin (1977) 88-95 MR0467734

[16] J W Milnor, J D Stasheff, Characteristic classes, Annals Math. Studies 76, Princeton Univ. Press (1974) MR0440554

[17] G Segal, Classifying spaces and spectral sequences, Inst. Hautes Études Sci. Publ. Math. (1968) 105-112 MR0232393

[18] G Segal, Configuration-spaces and iterated loop-spaces, Invent. Math. 21 (1973) 213221 MR0331377

[19] G Segal, Categories and cohomology theories, Topology 13 (1974) 293-312 MR0353298

[20] G Segal, $K$-homology theory and algebraic $K$-theory, from: " $K$-theory and operator algebras (Proc. Conf., Univ. Georgia, Athens, Ga., 1975)”, (B B Morrel, I M Singer, editors), Lecture Notes in Math. 575, Springer, Berlin (1977) 113-127 MR0515311

[21] G Segal, The definition of conformal field theory, from: "Topology, geometry and quantum field theory", (U Tillmann, editor), London Math. Soc. Lecture Note Ser. 308, Cambridge Univ. Press (2004) 421-577 MR2079383

[22] S Stolz, P Teichner, What is an elliptic object?, from: "Topology, geometry and quantum field theory", (U Tillmann, editor), London Math. Soc. Lecture Note Ser. 308, Cambridge Univ. Press (2004) 247-343 MR2079378

[23] S Stolz, P Teichner, Supersymmetric field theories and integral modular functions, Preprint (2007) Available at http://www.nd.edu/ stolz/preprint.html

[24] V S Varadarajan, Supersymmetry for mathematicians: an introduction, Courant Lecture Notes in Math. 11, New York Univ. Courant Inst. (2004) MR2069561

[25] E Witten, The index of the Dirac operator in loop space, from: "Elliptic curves and modular forms in algebraic topology (Princeton, NJ, 1986)", (P S Landweber, editor), Lecture Notes in Math. 1326, Springer, Berlin (1988) 161-181 MR970288

School of Natural Sciences, Institute for Advanced Study

1 Einstein Drive, Princeton NJ 08540, United States

markert@ias.edu

Received: 24 May 2007 Revised: 25 November 2009 